\title{
Portfolio Policies with Stock Options
}

This version: May 2, 2008

First Version: January 2008

\begin{abstract}
We study the partial equilibrium portfolio optimization problem for a myopic CRRA investor who can trade options on individual stocks. Applying the parametric portfolio approach of Brandt, Santa-Clara, and Valkanov (forthcoming) to derivatives we show that options characteristics (such as implied volatility and IV smile skew) convey information about the mispricing in the option portfolios. We take the data on all US-traded options to build characteristic-based factor portfolios of options. An investor uses them in addition to the market portfolio and Fama and French (1992) factors in her utility maximization. Surprisingly, portfolios based on the IV smile skew turn out to be less important than IV-based portfolios, and factor portfolios from call options are in general more interesting for an investor than the factors from puts. Market frictions in the form of stock shortsale constraints are compensated by the use of options, and having options with no stock shortsales allowed may even be better than having only stocks with shortsales permitted. Monthly rebalancing leads to extreme transaction costs for an investor facing the full bid-ask spread, providing a limits to arbitrage interpretation of the documented mispricing in the option portfolios.
\end{abstract}

Keywords: stock options, portfolio analysis, hedge fund policy, implied volatility, skew risk

JEL: C21, G13, G14 


\section{Introduction}

Individual stock options represent an important class of financial assets. In one-factor models like Black and Scholes (1973) and Merton (1973) the plain option is just a leveraged position in underlying with an implicit insurance against adverse stock movements. In reality options violate assumptions of one-factor models and fit rather multi-factor models with stochastic volatility (Heston (1993), Bates (2000), among others) and/ or jumps (Bates (1988), Bakshi, Cao, and Chen (1997) and others). In this case we have several possible scenarios: an option-specific factor is systematically priced, i.e. an option with higher exposure to such a factor bears a systematically different risk premium in its return, an option-specific factor is not priced and the associated risk is not interesting for an investor, or an option-specific factor is not systematically priced, but an investor would get an exposure to it due to an irrational but long-lasting mispricing ${ }^{1}$. It is important to be able to isolate such factors empirically, i.e. to find the proxies (instrumental variables) that allow us to approximate the factors from the options' payoff space.

In this paper we attempt to solve this problem via maximizing the CRRA utility for a myopic investor having access to the US exchange-traded individual options and underlying stocks ${ }^{2}$. Our main contribution is that we use a very extensive dataset of stock options (on about 5,000 underlyings) to bridge and extend two strands of literature on empirical option pricing: research that deals with preference-free analysis of the option pricing anomalies, and preference-based portfolio analysis with derivatives. We identify the characteristic-based factors from the options return space that benefit an investor with the CRRA utility. We are also able to interpret the optimal factor combinations intuitively in the form of commonly used trading strategies.

Our second contribution is an application of the parametric portfolio policy approach (Brandt, Santa-Clara, and Valkanov (forthcoming)) in the setup with individual options. We show that it is a powerful tool to build factors from the existing payoff space and to use them in the investor's optimization problem. It allows us to reduce the dimensionality of the optimization problem from a large number of assets to a significantly smaller number of factors, or characteristics, that we use to build those factors.

The bulk of recent empirical option pricing studies concentrate on index options. Bondarenko (2003), Constantinides, Jackwerth, and Perrakis (forthcoming), Bakshi and Kapadia (2003b) among others document violations of one-factor model assumptions and confirm the negative sign of the variance risk premium for index options. The limited research on individual stock options has not come to an agreement on the sign and nature of variance risk premia yet, and we hope to set guidelines by providing a new evidence on stock option-based factors. Bakshi and Kapadia (2003a) study 25 individual equity options and find that their prices embed a negative market volatility risk premium, but the magnitude of this risk premium is small. Driessen, Maenhout, and Vilkov (2007) look at options on all S\&P100 component stocks and do not find economically significant

\footnotetext{
${ }^{1} \mathrm{~A}$ considerable mispricing may exist for a long time due to transaction costs, and this limits of arbitrage argument is consistent with the empirical results in Section 6.3

${ }^{2}$ The form of preferences is not a crucial assumption in our partial equilibrium setup: we use a specific utility function mainly in order to compare the portfolio return distributions to get the optimal one. We could have compared the moments of distributions directly, but it would be trickier to get the optimal ordering of portfolio policies.
} 
returns of those in excess of one-factor model. Carr and $\mathrm{Wu}$ (forthcoming) investigate the synthesized variance risk swaps for 35 individual securities. They find a large crosssectional variation in variance risk premia, and conclude that the majority of the market variance risk premium is generated by an independent variance risk factor. Goyal and Saretto (2007) study short-term ATM options on individual stocks and find an economically important source of mispricing in the options implied volatilities. They conclude that zero-cost trading strategies in options constructed on the base of variance risk premium proxy produce a significant average monthly return. We extend this literature in two dimensions. First, we confirm that on an economy-wide scale with all exchange traded stock options, the options on individual stocks are not simple leveraged substitutes (with insurance) for stock risk factors, and that option returns are partly driven by independent risk factors. Second, we show that risk factors for options of different moneyness/ maturity/ type profiles may not be correlated very much, and this opens a Pandora's Box with respect to modeling options' dynamics. What we fail to show for now is that these factors are indeed systematic, and this important question is left for further research.

Previous research shows that options may indeed represent a lucrative asset class for different investors. Liu and Pan (2003) find that access to derivatives influences optimal stock holdings, improves the portfolio performance and plays an important role in utility maximization for non-myopic investors. Driessen and Maenhout (2007) study empirically the problem of portfolio choice with OTM puts and ATM straddles investments. They consider several types of investors and find that all of them should optimally have short positions in the derivatives to earn the jump and volatility risks premia. Haugh and Lo (2001) use the idea of the Black-Scholes option pricing model to construct buy-and-hold portfolios consisting of bonds, stocks and options. They are not able to analyze a large number of options, but find that options are interesting for an investor under various underlying models and types of preferences. Most of the research on portfolio choice works with stocks, bonds and index options. Our analysis is different in two dimensions. First, we use a much larger dataset of individual options, and second, we do not allow investors to take outright (i.e. just long or short) positions in options. We build factor portfolios from the options assigned to several asset classes (with similar maturity/ moneyness/ option type profile), and each of those portfolios represents an approximately market delta neutral long-short spread trade.

A direct maximization of an investor's utility who also invests in derivatives is a very complex problem. A great amount of research is focused now on finding the ways to simplify the portfolio optimization problem. Instead of focusing on the properties of distribution of returns of the assets in the portfolio, we take the direction similar to Brandt (1999), Ait-Sahalia and Brandt (2001) and Brandt, Santa-Clara, and Valkanov (forthcoming). Brandt (1999) solves the problem of finding portfolio weights such that expectation of marginal utility is equal to zero, conditional on forecasting variables and without making any assumptions on how returns depend on those variables. Ait-Sahalia and Brandt (2001) argue that the best approach would be to choose variables that are able to predict portfolio weights directly rather than first to try to predict returns. Using a semiparametric approach to construct an index from the default spread, the log of the dividend-to-price ratio, the term spread, and the momentum, they look for an optimal portfolio in terms of this index composition.

Building on the previous results we perform our analysis in the following steps. First, 
we select the characteristics of the options and underlying securities that may convey information about the priced risk factors. For stocks those are traditionally size, book-tomarket (Fama and French (1992)), and momentum (Carhart (1997)). For options we use the level of implied volatility and the skew size as characteristics ${ }^{3}$.

Second, we divide all options into the standard asset classes by moneyness (OTM and ATM), time to maturity (short- and long-dated) and option type (calls and puts). For each asset class we create quantile based factor portfolios for each of the two characteristics ${ }^{4}$. Interestingly, many pairs of the resulting factor returns have very low correlation, and cannot be treated as substitutes. Potentially it can lead us to a hypothesis that puts and calls, for example, are in part driven by different risk factors. We also estimate the risk premia on those factors in stocks' and options' portfolios. It turns out that many of identified factors indeed contribute systematically to option returns, while not having significant effect on stock returns.

Third, we use the parametric portfolio approach of Brandt, Santa-Clara, and Valkanov (forthcoming) and work with portfolio weights in a form of a function of the assets' characteristics. Effectively, we make our portfolio weights a function of 1) the assets' weights in factor portfolios (as parameter), 2) the decision variable $\theta$ that represents the weight of each factor portfolio. Solving the investor's optimization problem for $\theta$, we can find the weights of the underlying assets (if we need to). Surprisingly, it turns out that for a CRRA investor most interesting factors are derived from implied volatility as a sorting variable, and many interesting factor combinations are built using call options (while most options' mispricing results in the literature are related to put options).

The paper proceeds as follows. In the next section we briefly discuss the general ideas of the parametric portfolio policies approach of Brandt, Santa-Clara, and Valkanov (forthcoming) and then apply these ideas to a portfolio optimization problem with derivatives. In section 3 we describe our data and discuss how we prepare it for further analysis applying various filters and aggregation. In section 4 we talk about the intuition behind choosing particular asset characteristics to build factors, show how we create the factor portfolios and elaborate on our expectations from portfolio optimization with those factors. In section 5 we explore the solution to the investor's problem. We show the optimal trading strategies and try to estimate risk premia on option-based factors. In section 6 we introduce several extensions and also discuss the impact of market frictions on the investor's actions. Finally, section 7 concludes.

\section{Parametric Portfolio Policy}

In this section we discuss the general idea of the parametric portfolio choice methodology, and show the formal setup of the optimization problem. It turns out that the approach

\footnotetext{
${ }^{3}$ As the implied volatility (IV) measure we take the constant volatility from the fitted binomial tree Cox, Ross, and Rubinstein (1979) with discrete dividends for American options and the usual Black and Scholes (1973) IV for European options. As the skew measure we use the difference between the IV of the OTM and ATM options

${ }^{4}$ We use characteristics as conditioning variables, i.e. we condition the factor portfolio composition in the month $i$ on the values of characteristics in the previous month $i-1$.
} 
allows for an easy application of the investor's problem within large universe of assets whose dynamic is driven by a number of common factors.

\section{$2.1 \quad$ General Idea}

Following Brandt, Santa-Clara, and Valkanov (forthcoming), we model portfolio weights directly as a function of a set of asset characteristics. A representative investor holds the market and then optimizes her final portfolio composition with respect to the specified factors derived from the asset returns and respective characteristics. We should note that in this paper we limit ourselves to linear factors, though it has been shown (Jones (1996)) that option returns rather follow a non-linear factor structure.

The average utility (from consuming the portfolio return each period) over a given time frame is an objective function of an investor, and it is maximized over the weights of factor portfolios in the total portfolio, or, in other words, over the loadings (denoted by $\theta$ - constant over time) on the assets' characteristics.

This scheme effectively decreases the dimension of the optimization problem from a large number of assets in the investor's universe to a relatively small number of factors. An important feature of this approach is that it is very general and allows for easy extensions. The inclusion of new factors merely represents a problem of computational capacity.

Brandt, Santa-Clara, and Valkanov (forthcoming) suggest to apply the parametric portfolio policy approach to bond or currency portfolios, using the characteristics of these assets as choice variables. Instead, we will add derivatives to the assets universe of a CRRA investor, i.e we will explore the implications of giving the investor access to the optionsbased factors. Interesting issues include the choice of factors maximizing the average utility, and interaction between the choice of stock- and option-based factors.

Next, we look at the formal setup of investor's optimization problem in the parametric portfolio approach.

\subsection{The Model}

Consider investor who wants to form her portfolio using individual stocks and options. Every month $i$ there are $J_{i}^{s}$ stocks in the economy and $J_{i}^{d}$ derivatives, so that the total number of assets equals $J_{i}=J_{i}^{s}+J_{i}^{d}$. Investor maximizes her conditional utility from consuming the portfolio return for each time period (month) $i$ :

$$
\max _{\left\{\omega_{i, j}\right\}_{j=1}^{J_{i}}} \mathrm{E}_{i}\left[u\left(r_{i+1, p}\right)\right]=\mathrm{E}_{i}\left[u\left(\sum_{j=1}^{J_{i}} \omega_{i, j} r_{i+1, j}\right)\right] .
$$

Here $\omega_{i, j}$ is the weight of asset $j$ at time $i, r_{i, p}$ is the portfolio return at time $i$ and $r_{i, j}$ is the periodic return of asset $j$ at time $i$.

We follow and extend the parametric approach of Brandt, Santa-Clara, and Valkanov (forthcoming) and construct portfolio weights $\omega_{i, j}$ for stocks and derivatives as a function of their characteristics $\omega_{i, j}=f\left(\bar{\omega}_{i, j}, x_{i, j}^{s}, x_{i, j}^{d}, \theta\right)$, where 


$$
f\left(\bar{\omega}_{i, j}, x_{i, j}^{s}, x_{i, j}^{d}, \theta\right)=\left(\bar{\omega}_{i, j}, \frac{1}{J_{i}^{s}} \theta^{T} x_{i, j}^{s}, \frac{1}{J_{i}^{d}} \theta^{T} x_{i, j}^{d}\right),
$$

where $\bar{\omega}_{i, j}$ is the weight of asset $j$ at time $i$ in the value-weighted market portfolio, $x_{i, j}^{s}$ is the vector of characteristics of stock $j$ at time $i, x_{i, j}^{d}$ is the vector of characteristics of derivative $j$ at time $i$, and $\theta$ is the vector that gives loads on the characteristics and has to be estimated.

As we use the same vector $\theta$ for stocks and derivatives in equation (2), then formally the following should be true: $\operatorname{dim}(\theta)=\operatorname{dim}\left(x_{i, j}^{s}\right)$ and $\operatorname{dim}(\theta)=\operatorname{dim}\left(x_{i, j}^{d}\right)$. We construct vectors $x_{i, j}^{s}$ and $x_{i, j}^{d}$ to have the same dimensions as $\theta$ and with zeroes on the last $\operatorname{dim}(\theta)-K^{s}$ elements of $x_{i, j}^{s}$ for the stocks' characteristics, where $K^{s}$ is the number of factors considered for stocks. Similar for derivatives' characteristics: the first $\operatorname{dim}(\theta)-K^{d}$ elements of vector $x_{i, j}^{d}$ are zeroes, $K^{d}$ is the number of factors considered for derivatives:

$$
\begin{aligned}
& x_{i, j}^{s}=(x_{i, j}^{s, 1}, \ldots, x_{i, j}^{s, K^{s}}, \underbrace{0, \ldots, 0}_{\operatorname{dim}(\theta)-K^{s}})^{T}, \\
& x_{i, j}^{d}=(\underbrace{0, \ldots, 0}_{\operatorname{dim}(\theta)-K^{d}}, x_{i, j}^{d, 1}, \ldots, x_{i, j}^{d, K^{d}})^{T}
\end{aligned}
$$

and the following holds: $\operatorname{dim}(\theta)=K^{s}+K^{d}$.

Note, that $\theta$ is chosen to be constant through the time, it means that portfolio weights are time dependent only to the extent of the time dependency of $\bar{\omega}_{i, j}$ and the characteristics of stocks and derivatives.

If we define the market portfolio as the benchmark, then the terms $\frac{1}{J_{i}^{s}} \theta^{T} x_{i, j}^{s}$ and $\frac{1}{J_{i}^{d}} \theta^{T} x_{i, j}^{d}$ can be considered as deviations from the benchmark portfolio. We standardize stocks' and derivatives' characteristics so that their cross-sectional distribution becomes stationary. As a result the cross-sectional means $\frac{1}{J_{i}^{s}} \sum_{j=1}^{J_{i}^{s}} \theta^{T} x_{i, j}^{s}$ and $\frac{1}{J_{i}^{d}} \sum_{j=1}^{J_{i}^{d}} \theta^{T} x_{i, j}^{d}$ are zero, compatible with an interpretation of the factor portfolio investment as deviation from the benchmark portfolio. The following equation holds by construction:

$$
\sum_{j=1}^{J_{i}} f\left(\bar{\omega}_{i, j}, x_{i, j}^{s}, x_{i, j}^{d}, \theta\right) \cdot 1_{3 \times 1}=\sum_{j=1}^{J_{i}}\left(\bar{\omega}_{i, j}+\frac{1}{J_{i}^{s}} \theta^{T} x_{i, j}^{s}+\frac{1}{J_{i}^{d}} \theta^{T} x_{i, j}^{d}\right)=1,
$$

where $1_{3 \times 1}$ is a vector-column of ones. We denote $r_{i+1, j}=\left(r_{i+1, j}^{s}, r_{i+1, j}^{s}, r_{i+1, j}^{d}\right)^{T}$, where $r_{i+1, j}^{s}$ and $r_{i+1, j}^{d}$ are returns at time $i+1$ on stock $j$ and derivative $j$ respectively.

Since $\theta$ does not depend on time, instead of maximizing investor's conditional utility in equation (1), it is enough to maximize her unconditional (or mean myopic) utility over $\theta$ :

$$
\begin{gathered}
\left(\frac{1}{T} \sum_{i=0}^{T-1} u\left(r_{i+1, p}\right)\right)=\frac{1}{T} \sum_{i=0}^{T-1} u\left(\sum_{j=1}^{J_{i}}\left(f\left(\bar{\omega}_{i, j}, x_{i, j}^{s}, x_{i, j}^{d}, \theta\right) \cdot r_{i+1, j}\right)\right) \\
=\frac{1}{T} \sum_{i=0}^{T-1} u\left(\sum_{j=1}^{J_{i}}\left(\bar{\omega}_{i, j}+\frac{1}{J_{i}^{s}} \theta^{T} x_{i, j}^{s}+\frac{1}{J_{i}^{d}} \theta^{T} x_{i, j}^{d}\right) r_{i+1, j}\right) .
\end{gathered}
$$


We do not impose constraints on $\bar{\omega}_{i, j}$ yet, so that short selling is allowed. We study the implications of the shortsale constraints below in Section 6.1.

We consider an investor with CRRA utility function

$$
u\left(r_{i+1, j}\right)=\frac{\left(1+\bar{\omega}_{i, j} r_{i+1, j}^{s}+\frac{1}{J_{i}^{s}} \theta^{T} x_{i, j}^{s} r_{i+1, j}^{s}+\frac{1}{J_{i}^{d}} \theta^{T} x_{i, j}^{d} r_{i+1, j}^{d}\right)^{1-\gamma}}{1-\gamma} .
$$

To solve the maximization problem (3) we apply Generalized Method of Moments (GMM). The moment conditions for the stocks' characteristics only are the following:

$$
\frac{1}{T} \sum_{i=0}^{T-1}\left[\frac{\partial u\left(r_{i+1, p}\right)}{\partial \theta_{k}}\right]=\frac{1}{T} \sum_{i=0}^{T-1}\left[u^{\prime}\left(r_{i+1, p}\right) \cdot \frac{1}{J_{i}^{s}} \sum_{j=1}^{J_{i}} x_{i, j}^{s, k} r_{i+1, j}\right]=0
$$

where $\theta_{k}$ for $k \in K^{s}$ is the weight of $k$-th characteristic of stocks in the portfolio, where the $k$-th characteristic of stock $j \in J_{i}^{s}$ at time $i$ is equal to $x_{i, j}^{s, k}$. Moment conditions for derivatives' part can be defined in the similar manner. The Hessian matrix of second derivatives in the case when $l \in K^{s}$ and $m \in K^{d}$ is equal to

$$
\frac{1}{T} \sum_{i=0}^{T-1}\left[\frac{\partial u^{2}\left(r_{i+1, p}\right)}{\partial \theta_{l} \partial \theta_{m}}\right]=\frac{1}{T} \sum_{i=0}^{T-1}\left[u^{\prime \prime}\left(r_{i+1, p}\right) \cdot\left(\frac{1}{J_{i}^{s}} \sum_{j=1}^{J_{i}} x_{i, j}^{s, l} r_{i+1, j}\right) \cdot\left(\frac{1}{J_{i}^{d}} \sum_{j=1}^{J_{i}} x_{i, j}^{d, m} r_{i+1, j}\right)\right] .
$$

We analyze the significance of the estimated $\theta$-parameters and utility gains from adding the new factors into the base case portfolio optimization in order to test if inclusion of the new factor improves the investment strategy.

Our benchmark portfolio is the market. We can rewrite the returns on the optimal portfolio in the following way:

$r_{i+1, p}=\sum_{j=1}^{J_{i}} \bar{\omega}_{i, j} r_{i+1, j}+\sum_{j=1}^{J_{i}}\left(\frac{1}{J_{i}^{s}} \theta^{T} x_{i, j}^{s}\right) r_{i+1, j}+\sum_{j=1}^{J_{i}}\left(\frac{1}{J_{i}^{d}} \theta^{T} x_{i, j}^{d}\right) r_{i+1, j}=r_{i+1, m}+r_{i+1, h}^{s}+r_{i+1, h}^{d}$,

i.e. it is the sum of the return on the market portfolio and of the return on a zero-cost portfolio that consists of two parts - equity $\left(r_{i+1, h}^{s}\right)$ and derivatives $\left(r_{i+1, h}^{d}\right)$. It is possible to interpret the problem (1) as a utility maximization of an investor who already holds the market and wants to invest in a (market-neutral) derivatives hedge fund.

\section{Data Description}

As assets for the portfolio selection problem of an investor we take individual stocks and plain vanilla options written on them. We restrict the universe of US stocks to those that have traded options within 1996 - 2004 time frame. To construct factor portfolios we use a number of company- and asset-related characteristics. The data processing procedures, i.e. initial selection, various filters we apply and variables we construct, are described in detail for stocks and options separately. 


\subsection{Stock Data}

We use Compustat as the main source for quarterly company-related data, and CRSP for information on monthly and daily stock returns and shares outstanding each month. Selecting only the US stocks with traded options, we end up with 4964 stocks and initially download the data for them from January 1995 to December 2004. We will use the following notation: $i \in\{1, \ldots, 108\}$ - time index and $j \in\{1, \ldots, 4964\}$ - firms' index.

We follow Brandt, Santa-Clara, and Valkanov (forthcoming) in forming the monthly stock factors from Compustat/CRSP variables. The exact definition of those variables can be found in Appendix A. From Compustat we get the values for assets, liabilities, common and preferred stocks and deferred taxes. If the price of a common stock does not exist in Compustat, we take it from CRSP. In the Compustat table returns are given monthly, and the rest of variables are quarterly. To get monthly data we apply linear interpolation of quarterly values, and we do so for assets, liabilities, preferred stock value and deferred taxes. As a result we get for these variables time series of 108-month (from January 1996 to December 2004) for 4964 stocks and use them to form the appropriate stock characteristics each month.

We define book equity as equal to total assets minus liabilities plus balance sheet deferred taxes and investment tax credits minus preferred stock value. Market equity is equal to price per share times shares outstanding. We omit observations with negative market or book equity values. Book-to-market (BTM) then is equal to the log of book equity divided by market equity. Size characteristic (ME) is computed as the log of market equity. We also calculate momentum each month for all available stocks on a rolling basis using the monthly returns over the past year as $M O M_{i}=\prod_{k=i-13}^{i-2}\left(1+r_{p, k}\right)$. For the 1996 year momentum we use monthly returns for 1995-1996 from CRSP.

\subsection{Options Data}

We use OptionMetrics to get monthly data for put and call options from January 1996 until December 2004. Since we already restricted the universe of stocks to those having options, it is not surprising that we have options on the same number of underlyings as the number of stocks, i.e. on 4964.

We work with raw data, i.e. with best bid and ask closing quotes rather than the interpolated volatility surfaces constructed by OptionMetrics. We select all options having 10 to 180 days to maturity, and then we discard options with zero open interest or with zero bid prices. To eliminate evident pricing errors we also remove the options with implied volatilities higher than $100 \%$.

We are interested in monthly holding period returns, and we construct those taking into account American feature of traded stock options. To calculate the return $r_{i, j}^{o}$ for a given option $o$ on underlying $j$ in month $i$ we use the price of that options at the end of the previous month and compare it with the value of the option at the end of a given month. The value of an option is the maximum between its price and the value of the immediate exercise.

Using different prices we also make an attempt to study the effect of the transaction costs on the portfolio policy. For the returns with zero transaction costs we use mid quotes 
as prices (i.e. (best bid +best offer)/2). For returns with transaction costs we take as initial price the best bid for a short position in options and the best offer for a long position. Then we close those positions on the other side of the bid-ask spread at the end of the month, i.e for the short position we take the max(best offer, value of immediate exercise) that is effectively equivalent to best offer and for the long position then we take the max(best bid, value of immediate exercise).

Usually a stock in our dataset has several options outstanding on a given day with various strikes, expiration dates, and types (put or call). Dealing with each option separately is quite hard as they are often not comparable cross-sectionally along moneyness and maturity dimensions. To standardize the choice of options for an investor we construct three moneyness $^{5}$ and two maturity buckets (corresponding to $[0.85,0.95],(0.95,1.05]$ and $(1.05,1.15]$ moneyness levels and $[10,90],(90,180]$ days to maturity) for calls and puts appropriately. Then we sort all options into corresponding buckets and aggregate options on one underlying into one security. We treat each bucket as the investable asset class with its calculated implied volatility and return. For the return and volatility calculations on aggregated options we assume equally weighted investments in all options on the same underlying assigned to a given bucket at a given time. On average there are about 200 aggregated options in each bucket at each point in time.

\section{Factors and Predictions}

The idea of parameterizing portfolio weights is a simplification of a general portfolio optimization problem. Of course, an important issue is the choice of factors, with respect to which we perform the optimization. Our approach is based on the features of stocks and derivatives markets and existing literature that tries to simplify investor's optimization problem and to go around the task of modeling the distribution of portfolio returns.

\subsection{Choice of Characteristics}

For the stocks the choice of characteristics is relatively straightforward and is based on previous research. We consider size (market capitalization), book-to-market ratio (Fama and French (1992)) and momentum (Carhart (1997), Daniel and Titman (1997), Jegadeesh and Titman (2001) among others). We regard this choice as a base case and add a number of derivatives with their respective characteristics (read, factors) to the investor's investment opportunity set.

Brandt, Santa-Clara, and Valkanov (forthcoming) investigate how the behavior of an investor would change if she considers interactions between stock-based factors. Instead we concentrate on the factors that stem from derivatives' characteristics. We expect them to improve the performance of an investor's portfolio.

In an extension we also look at the effect of the stock shortsale constraints (sections 6.1) and at the stock's liquidity factor (section 6.2). We also test the validity of the results for portfolio with derivatives where an investor is paying the full bid-ask spread at each

\footnotetext{
${ }^{5}$ Where moneyness is defined for each options as a relation of strike $(K)$ to spot price $(S)$, i.e. $\frac{K}{S}$.
} 
rebalancing date (section 6.3). We do not consider a transaction costs factor explicitly, although it might have interesting interaction effects with other characteristics and serve as a proxy for the derivatives' liquidity factor. We leave this issue for later research.

To choose the options' characteristics we simply regard options as at the derivatives written on the underlying stocks. Under the assumption of an Ito process for the stock $S$, driven by a (multidimensional) Brownian motion $d W_{t}^{S}$ and possibly a jump process $d J_{t}$

$$
\frac{d S_{t}}{S_{t}}=\left(\mu-\lambda E\left[\xi_{t}\right]\right) d t+\sigma_{t} d W_{t}^{S}+\xi_{i} d J_{i}
$$

the derivative security return will be driven by the same Brownian motion and the same jump process. In addition, if the diffusion coefficient $\sigma_{t}$ by itself is stochastic, i.e. its dynamics can be described by an SDE as for example in Heston (1993) model,

$$
d \sigma_{t}^{2}=\kappa\left(\theta-\sigma_{t}^{2}\right) d t+\varsigma \sigma_{t} d W_{t}^{\sigma}, d W_{t}^{S} d W_{t}^{\sigma}=\rho_{t} d t
$$

the SDE for derivative asset inherits the factors driving diffusion coefficient (and correlation $\rho_{t}$ between the Brownians if it is stochastic as well) by the simple application of Ito's lemma. The same holds true for jumps: option's SDE inherits the jump process from the stock, and furthermore if the jump size/intensity are stochastic, their driving processes will show up in the SDE for derivative security $Q$ as well.

$$
\begin{aligned}
Q_{s}-Q_{0} & =\int_{0}^{s}\left\{\frac{\partial Q_{t}}{\partial t} d t+\frac{\partial Q_{s}}{\partial S_{t}} d S_{t}+\frac{1}{2} \frac{\partial^{2} Q_{t}}{\partial S_{t}^{2}}\left[d S_{t}\right]^{2}+\frac{\partial Q_{t}}{\partial \sigma_{t}^{2}} d \sigma_{t}^{2}+\frac{1}{2} \frac{\partial^{2} Q_{t}}{\partial\left(\sigma_{t}^{2}\right)^{2}}\left[d \sigma_{t}^{2}\right]^{2}+\frac{\partial^{2} Q_{t}}{\partial S_{t} \partial \sigma_{t}^{2}}\left[d S_{t} d \sigma_{t}^{2}\right]\right\} \\
& +\sum_{0<\tau \leq t}\left\{Q(S(\tau))-Q\left(S\left(\tau_{-}\right)\right)\right\} .
\end{aligned}
$$

Thus, the option returns in this setup will be driven by underlying in a leveraged fashion as well as by stochastic volatility and/or jump processes. If an investor first buys the market portfolio, and then only adjusts her appetite for direct stock-based factors like ME, BTM and MOM, she does not normally need options. However, after taking the risks represented by the stock's Brownian motion $W_{t}^{S}$, she may want to go for pure volatility/jump risk factors and hence build up positions in options. We recognize this and build our factors for derivatives using a proxy for volatility and jump risks priced in options ${ }^{6}$. Reasonable proxies for this purpose are the implied volatility from the BlackScholes model (Black and Scholes (1973)) for European options or from the binomial tree (Cox, Ross, and Rubinstein (1979)) for American options, and the skew size measure. Implied volatility level and volatility smile across moneyness and time convey information on factors affecting option returns directly and through leveraged underlying's continuous risk factors. This intuition is confirmed by several studies of the option markets.

Carr and $\mathrm{Wu}$ (forthcoming) show that the part of the market risk factor is correlated with the return variance, and that ME, BTM and MOM risk factors can explain only a small portion of the documented variance risk premia. The authors conclude that the

\footnotetext{
${ }^{6}$ As we discuss below in Section 4.3, our option-based factors are approximately delta-neutral by construction, and hence we indeed deal with the variance risk or small changes in underlying and can hope for some variance risk factors identification that are not priced in stocks.
} 
market variance should be largely driven by risk factors independent of those driving the underlying returns. Thus, we expect other factors, probably built on the existing assets like options, to be able to explain the returns of derivative assets.

The CAPM fails to explain why small and value stocks tend to be underpriced. Pietro and Vainberg (2006) study how firm characteristics like size and book-to-market relate to equity option expensiveness and check if these characteristics influence the pricing of systematic variance risk in the equity options market. They find that under the same level of expected realized variance under physical measure, options on small stocks are more expensive than options on large stocks and that options on value stocks are more expensive than options on growth stocks. Authors claim that overestimation of risk on small and value stocks leads investors to demand higher rates of returns on those types of stock and propose to study also the influence of momentum earnings on the options pricing. Implied volatility tend to be higher for small firms, as well as for high book-tomarket ratio firms. Duan and Wei (forthcoming) show that a higher amount of systematic risk leads to a higher level of implied volatility and a steeper slope of the implied volatility curve. Following these findings, we can expect some interaction between IV and skew size factor on the one side, and BTM, ME and MOM factors for stocks, on the other side.

Our choice of the second characteristic of the derivative assets is additionally supported by the work of Bates (2001), who views the options markets as an insurance market for crash risk. Deriving myopic portfolio allocation he concludes that equity and crash insurance are complements in the event of large negative jumps on the market. Branger, Breuer, and Schlag (2006) look at the optimal exposures to volatility and jump factors in a semi-simulated environment, and confirm that an investor with CRRA utility optimally chooses to take exposure to jump and volatility risks under discrete rebalancing conditions.

Thus, we find it reasonable to pick as characteristics for the derivative assets the level of implied volatility and a measure of the smile skew, calculated as the difference between the implied volatility for OTM and ATM options. We call it a skew or a crash factor, though it corresponds to a crash factor only for put options, and for call options it's rather a factor of positive jump or of an effect of stochastic volatility.

\subsection{Building Factor Portfolios}

We define an asset class as the collection of financial instruments with a predetermined payoff, maturity and moneyness profile. For example, we can define a class of all out-of-themoney call options with maturity between 10 and 90 days and so on. As described above, we construct three moneyness and two maturity buckets (corresponding to [0.85, 0.95], $(0.95,1.05]$ and $(1.05,1.15]$ moneyness level intervals and $[10,90],(90,180]$ days to maturity intervals) for calls and puts appropriately. Each of the buckets represents an investable asset class.

After we have defined the characteristics for a given asset class, we start building factors. For each factor portfolio we would like to get equally weighted exposure to the assets in extreme quantiles, with positive sign in extreme upper quantile, and with negative sign in the lower one. For every date $i$ we form three quantiles for the characteristics and assign the values $-1,0$ and +1 to lower, middle and upper thirds. These values correspond

to the $x_{i, j}^{a s s e t}$ 's in the portfolio weight definition (2). Then we divide each element of 
the vector $x_{i, j}^{s}$ (for stocks) and $x_{i, j}^{d}$ (for derivatives) by the number of elements in the corresponding quantile. By construction, those normalized dummies $\frac{x_{i, j}^{s}}{J_{i}^{s}}$ and $\frac{x_{i, j}^{d}}{J_{i}^{d}}$ sum up to 0 cross-sectionally (when the number of elements in the upper and lower quantiles is equal, which can easily be achieved). We treat then these normalized quantiles as asset weights for the factor portfolios corresponding to a given characteristic of a specified asset class. The decision variable $\theta$ manages our exposure to each factor portfolio, and the goal is to optimize the investor's utility with respect to $\theta$.

Thus, we define factor portfolios with respect to the characteristics of an asset class. It follows that in the given setup we limit the option strategies of investors to long/short positions in options of the same type (calls or puts), long/short synthetic forwards (when $\theta$ for the same moneyness and maturity calls and puts is of a different sign, but has the same absolute value), and long/short strangles (when $\theta$ for the same moneyness and maturity calls and puts are of the same sign and same absolute value). Maturity differences also imply some horizontal spreads in forwards and strangles. In Tables 1 and 2 we provide examples of attainable strategies for different $\theta$ combinations. We do not include straddles or single options in our set of investable strategies as it has been done in the literature (e.g. in Driessen and Maenhout (2007)). Our main goal is to construct factor portfolios from options that improve the investor's asset allocation, and to test if the resulting factors bear a systematic risk premium in traded assets.

\subsection{Predictions and Expectations}

As discussed earlier, we start with the market portfolio and then maximize the investor's utility by using the enlarged investment opportunity set with a number of additional factors. For the stocks we take ME, BTM and MOM factors, and for options we create factors for each asset class based on implied volatility level and skew size.

Though we have a relatively short sample period, for the stock factors we do not expect to obtain results which differ from previous research. The investor would deviate from the market by investing in small stocks and finance this purchase shorting large cap stocks (Fama and French (1992), Fama and French (1996)), by buying value stocks and stocks outperforming the market in the past, and finance it by selling growth and stocks that did not perform well in the last 12 month (Jegadeesh and Titman (1993), Jegadeesh and Titman (2001), Carhart (1997)).

For options we have two characteristics - the level of implied volatility and the volatility smile skew measure - applied to twelve asset classes $(3 \times 2 \times 2=$ moneyness $\times$ maturity buckets for calls and puts). When we create factor portfolios for options, we build approximately delta-neutral positions as within each asset class we buy and sell options of about the same delta level. It makes our factor portfolio immune to small changes in the price of the underlying and thus roughly isolates the volatility risk factor.

From the literature we know that OTM and ATM options tend to be mispriced (or we face the peso problem as in Branger and Schlag (2005)) as respective asset classes with respect to standard asset pricing models (Constantinides, Jackwerth, and Perrakis (forthcoming), Bondarenko (2003), and an investor optimally sells those options (Driessen and Maenhout (2007) among others). Tables 3 and 4 provide statistics on all investable 
asset classes we created: for individual options the mean return is quite negative and the distribution has pronounced fat tails. Returns are positively skewed and very volatile. OTM options show more negative returns than ATM and ITM contracts. Table 5 shows the statistics for the option factor portfolios we created. Overall their distribution has very fat tails and is mostly negatively skewed; some of the factors should be interesting for investors (judging by the Sharpe Ratios with absolute magnitude above 1).

For our purpose it does not matter if the option group is mispriced with respect to some asset-pricing model, since we do not allow the investor to go short or long all options in an asset class. Instead, we are looking at the mispricing of options within one asset class with respect to the average for this class, and try to infer priced factors (for an investor with a given utility function) from this mispricing. For asset classes with fairly priced volatility and jump risks we still expect to see no systematic preference of one options group over the other, i.e. we would expect the $\theta$-estimates to be insignificant.

From all asset classes we select only those containing OTM and ATM options. ITM options effectively resemble the leveraged position in the underlying and we want to study the options having larger time value (relatively to the option price), i.e. higher compensation for the risks of the option seller. Thus we are left with 4 moneyness and maturity buckets, two for calls and puts respectively.

As we talk about volatility and skew risks (whatever their real sources are), it makes sense to sell options with higher volatility and skew characteristic values (it makes more sense to sell options with high risk premium on those factors, but under assumption that risk premium is proportional to the levels, we can treat levels of skew and volatilities as premium proxies).

\section{Results}

In this section we discuss the results and see if our previous intuition is confirmed by the empirical findings. First we look at the base case with only stock factors available, then we move to extensions with various option-based factors. It is important to keep in mind that the interpretation of the values, especially the sign of $\theta$ parameters depends on the ranking of the correspondingly sorted characteristic cross-sectionally for a given asset class. For example, for the stocks, negative $\theta$ means going short stocks with a high value of a given characteristic and long stock with a low value of the characteristic. For options positive $\theta$ means being long options with a high value and short options with a low value of the characteristic. If we take the level of implied volatility as characteristic for asset class containing short maturity ATM put options and we find positive optimal $\theta$, we will buy puts with a relatively high volatility and sell puts of this bucket with a relatively low volatility.

\subsection{Base Case}

We consider investment into equities as the base case, it means we buy the market and then we take care of three main stock factors: size (ME), book-to-market (BTM) and momentum (MOM). The results are in line with previous research and our intuition. 
Brandt, Santa-Clara, and Valkanov (forthcoming)(BSCV) obtain as optimal estimate for $\theta$ the vector:

$$
\left(\theta_{m e}, \theta_{b t m}, \theta_{\text {mom }}\right)=(-1.220,3.466,2.00),
$$

for the CRRA case with $\gamma=5$. For the same risk aversion we get $\theta=(-1.559,2.469,2.130)$. Taking into account different data used for estimations, we can say that our results are in line with previous research. An investor would deviate from the market by being long small stocks, value stocks, and past winners (and short large caps, growth and past losers respectively).

Adding the option-based asset classes and factors to the investor's investment opportunity set in the next subsection we investigate the appetite of an investor to volatility and skew risk factors.

\subsection{Stocks and Options}

We do not investigate options separately from stocks, since we hope to capture factors common to stocks and options through the ME, BTM and MOM factors.

Table 6 shows the time series correlations between the factor portfolios based on various asset classes. We should be careful in choosing the factor portfolio combinations for the optimization problem, since factors based on the same characteristic for different asset classes may be highly correlated. If we see that investors are taking opposite positions in those factors, we may face a collinearity bias. The good news is that the option-based factors are hardly correlated with the stock-based factors, so they do not seem to be substitutes for each other. The maximum absolute correlation is 0.38 , while the maximum correlation between the stock-based factors is much higher with a value of 0.72 between MOM and BTM. The factors based on calls and puts are close to independent between option types with maximum absolute correlation of 0.39 for all pairs, and we can freely mix them in the portfolio. We can also combine certain IV factors with skew factors for calls and puts, but it seems dangerous to include several IV factors for the same options type.

To identify interesting factor combinations we look at several measures shown in Tables 7-8 for different levels of risk aversion. First, after solving the maximization problem of an investor for the whole sample period from January 1996 to December 2004 (107 months), we get portfolio composition with optimal levels of $\theta$. For each optimal portfolio we calculate distributional characteristics of returns over the whole sample period, mean utility level and look at the utility gain from adding option-based factors to the base case. We discard the combinations that do not result in significant utility gain over the whole sample period ${ }^{7}$. To test the stability of estimations we also solve the optimization problem over the first $2 / 3$ of the sample period (i.e. 72 months) and compare the utility calculated with $\theta$ 's of the 72 month period to the utility over the remaining 35 months using the $\theta$ estimates of the first 72 months. We discard the factor combinations resulting in different mean utility levels over the last 35 months depending on the $\theta$ estimation period. From

\footnotetext{
${ }^{7}$ We test the significance of the utility gain by comparing the means of utility time series for a given factor combination and the base case for the whole sample period.
} 
the surviving combinations ${ }^{8}$ we select 10 for the exposition. This last choice is not fully ad hoc: we first take the combinations with as few option-based factors as possible, and it gives us 4 portfolios with 2 or fewer option-based factors and the rest with 4 or more. All these combinations are potentially interesting for further analysis as all of them result in significant positive $\alpha$ with respect to linear factor models (with FF and momentum factors $)^{9}$.

Surfing the tables, we can point out the following regularities. First, higher risk aversion leads to lower $\theta$ 's, i.e. to smaller deviations from the market portfolio. Overall this gives more modest returns and smaller risks expressed by standard deviation of the portfolio returns over time. Second, there are not many extreme positions with opposite signs in option factor portfolios, so collinearity does not seem to be a problem for the surviving combinations.

Looking broadly at the $\theta$ values, we can summarize the following findings. Contrary to the common belief, we do not find many significant $\theta$ 's based on skew (crash) characteristic of the options. If there is some dispersion in volatility/jump pricing for the selected asset classes, we can barely identify it using the skew size. We will be selling skew factor for longterm OTM calls, buying some of it for long-term ATM calls. It means that volatility/jump risk premia are relatively too high for long-term OTM calls with large skew and/or too low for same options with small skew. For long-term ATM calls the risk premia is relatively too high for low skew options. Interestingly that for put options the crash factor looks even worse: we get the best significance for short ATM puts, while the common belief is that short OTM puts' pricing is heavily driven by crash fears. In any case, crash factors never seem to be working alone, and show up normally in combination with the IV-based factors.

Implied volatility seems to be more important as the characteristic variable for most asset classes, and an inclusion of just one asset class IV factor can lead to a significant utility improvement over the base case (as we see in the combination 2 in Tables 7 and 8). With the IV characteristic for options at our disposal, we will be buying either short- or long-dated OTM calls' factor portfolios, and/or selling either short- or long-dated ATM calls' factor portfolios. Long position in short-dated OTM put factors also benefit an investor, while the long-dated puts-based portfolios do not contribute much.

It is hard to name the most important factor as obviously there is lots of interplay between them. The combinations with more factors included make the investor generally better off in terms of utility (surely if we do not take into account associated transaction costs from monthly rebalancing). Comparing calls and puts we come to the conclusion that there is more mispricing in the call based portfolios than in the put based ones. Using an only one ATM calls portfolio derived from IV as characteristics the investor significantly improves her utility, reaping an additional $3.77 \%$ certainty equivalent (expressed as percentage of the initial wealth) over the base case for the $\gamma=1.8$ (Table 8). Just two call-based portfolios as in combinations 4, 5, 6 work better for an investor than four

\footnotetext{
${ }^{8}$ Depending on risk aversion we have different number of valid factor combination passing all tests. For $\gamma \in\{1,1.8\}$ we have 25 and 23 combinations respectively. Full results can be provided upon request.

${ }^{9}$ Base case scenario also shows a positive $\alpha$, though it is based on the same 4 factors that we use for regression. It is due to difference in factors: in our investor optimization problem we use factors built from our stock sample, while in factor regressions we use ready-to-use factors from Kenneth R. French web-site.
} 
put-based portfolios as in the combination 3 .

Including all possible factors in an investor's optimization problem makes her even better off, but most $\theta$ 's end up being not significant (except for log utility case). Based on the informal analysis of how many times the theta for a given factor shows up significantly in the estimations ${ }^{10}$ we assign the trading signals to a given characteristic quantile. The results are given in the Table 9 , and they confirm our previous findings that call-based factors are more important for an investor. Within the calls the long-dated options give more interesting factor portfolios than short-dated ones: we have three strong signals and one without grade for long-dated calls vs. two strong and two weak for short-dated ones.

To give the reader some additional intuition on the option-based factors, in the Tables 1 and 2 we also provide an interpretation of pairwise factor combinations in the form of spread trading strategies. Most of them look plausible and are easy to construct, e.g. characteristic spread in strangles, where we buy (sell) strangles for all options with low value of characteristic and sell (buy) strangles for all options with high value of the same characteristic. The combination 5 in Table 8 is exactly this kind of strategy: we are buying short maturity strangles with high volatility and finance them by selling the strangles of the same maturity with low volatility. The combinations 4 and 6 in the same table represent a characteristic spread in vertical spreads, where we buy vertical spreads on calls with low volatility and sell them with high volatility (for long and short maturities respectively). The combination 2 is just a factor portfolio, and the others are hard to interpret. Thus, we use our initial factor portfolios as building blocks for more complex strategies and note that those strategies can also be used as building blocks to get us to a new level of complexity.

Following Pietro and Vainberg (2006) we can also expect interaction effects between the option and stock factors. In the current setup we can identify the option asset classes where the option-based factors are substitutes for the stock factors in the investor's problem. Size factor $\theta$ does not seem to fluctuate much across the combinations, and it always remains significantly negative for all risk aversion levels. The other two stock factor $\theta$ 's are less stable. The first $\theta$ responsible for BTM factor drops by most from the base case level with addition of short OTM puts IV factor, and does even lose significance for a number of combinations. The other $\theta$ corresponding to momentum also drops with the inclusion of the same OTM put IV factor, but not that significantly. It provides us with indirect evidence that there is a relation between the pricing of volatility in short-dates OTM puts and the value vs growth anomaly in stock returns. The correlation between the corresponding factors is quite low ( 0.23 for BTM and short OTM puts IV factor), so the relation must be non-linear. One way of looking into the issue deeper would be the direct estimation of the factor risk premium in stock returns, and this is left for further research. It can also be interesting to test if some simple non-linear functional dependency of carefully chosen factors gives us a reasonable explanation of documented interaction.

The above discussion is based on the results in an ideal environment for an investor, where she does not face any transaction costs in the form of bid-ask spread, nor has she any shortsale constraints on stocks. Liquidity of market for a given instrument may influence the investment strategy as well. In the following sections we also try to shed the

\footnotetext{
${ }^{10}$ Here we take into consideration all 23 surviving factor combinations for $\gamma=1.8$ and not only those presented in Table 8.
} 
light on the optimal portfolio with market frictions.

\subsection{Factor Premia Estimation}

As we have seen from interaction between stock and option-based factor portfolios in the previous section, and as has been noted in the previous research (Ang, Hodrick, Xing, and Zhang (2006), Xing, Zhang, and Zhao (2007) among others), parameters of the underlying's process (realized volatility or observed jumps as proxy for it) and its observed effect on options' characteristics (skew in the risk neutral probability distribution, or IV smile skew size as its proxy) can reveal to us important asset pricing factors, either systematic or resulting from a sustained mispricing. Pricing errors $(\alpha)$ in the portfolio return with respect to common linear factor (market, $3 \mathrm{FF}$, and $3 \mathrm{FF}+$ momentum) models confirms this intuition. As we see in Tables 7 and 8, each portfolio combination demonstrates a highly significant positive $\alpha$ over each of the generally accepted linear models.

We are going to estimate the risk premia on the factors derived from option asset classes with appropriate characteristics and to see if those factors are systematically priced in stocks and in options. There are several possibilities for the outcome: first, option-based factors may be priced in both stocks and options; second, they may be priced in options, but not priced in stocks, and third, they can be priced in either stocks or options, or in both, but the estimations may be not robust. The first two outcomes are interesting and both have important implications. The last outcome may be due to a short estimation period, or may hint at the sustained mispricing in the option factor portfolios. It would make option portfolios interesting for an investor, but they will not be loading on any systematic factors.

The first decision we have to make is what factors and what asset returns we take. We have seen in Table 6 that many factors for different asset classes are highly correlated, and there is little sense to estimate risk premium on all of them at the same time. The model will be definitely misspecified as options have non-linear factor structure (Jones (1996)), and it is not our purpose here to describe it completely. All we want to see if linear factors based on option characteristics bear a significant risk premium affecting option returns directly or through stock returns. We have seen in Tables 7-8 and 9 that the most interesting factor portfolios are based on IV level for OTM calls (both short and long-dated), IV level for ATM calls (again both maturities), on the skew size for long-dated OTM calls and IV level of short-dated OTM puts. Our working hypothesis would be that those factors represent priced systematic risk factors for option returns. We would expect the IV from OTM short puts factor to be priced in stocks though its interaction with the BTM factor.

For better diagnosis of what factors are important, we estimate risk premia for each option-based factor individually. We follow standard two-stage Fama and MacBeth (1973) procedure and include in factors three FF factors (Fama and French (1992)), momentum (Carhart (1997)) and one of the option-based factors. To be consistent with other asset pricing literature we use not the self-made factors, but rather download the ready-to-use ones from Kenneth R. French web-site. We use returns on all options in the defined asset 
classes $^{11}$, and all 4964 time series of stock returns used in the paper to form standard portfolios. We first build 3 portfolios for each of the option asset classes using the fullsample estimated option factor $\beta$ as a sorting variable. It gives us 36 derivatives portfolios. Then we build 30 portfolios from stocks, following the same procedure and using the estimated factor $\beta$ as a sorting variable. On the second stage we infer the factor risk premia with GLS (using the variance-covariance matrix of first stage residuals as weighting matrix as suggested by Shanken (1985) among others) from 36 option portfolios and 30 stock portfolios separately. In the Table 10 we show the estimated risk premia on market, three Fama-French and momentum factors, plus one selected option-based factor. In Table 11 we also show the summary results for estimated risk premium on option-based factors using 36 option and 30 stock portfolios, as well as the t-statistics calculated with Shanken (1992) correction in both cases.

We should be very careful in drawing any strict conclusions from the results: as we noted, the tests are definitely ill specified (as we can see from large significant $\alpha$ 's in each regression), the portfolio returns and factors are too volatile and the time series are rather short (only 107 months) to identify the factor risk premia with confidence. After we correct the second step regression standard errors for errors in estimated betas (due to Shanken (1992)), we hardly get any significance even for standard factors. The risk premia in stocks is not big enough in the first place to be able to split it into so many components using limited series length. In options the risk premia is bigger in magnitude (due to their leveraged nature), and we get enough significance even after all necessary standard errors' corrections. However, when we compare the calculated risk premia for option-based candidate factors with the time series mean of the respective factors in Table 5 , we see that the estimations are not nearly the level where they should be. Thus, we should either increase the sample length or come up with some other testing method.

Our exercise still delivers some interesting results concerning the standard linear factors we used in the estimation. The risk premia estimations for SMB and MOM factors are mixed, while the estimations for the market and for HML risk premia are very stable, and the HML risk premium is everywhere negative. Mean value of the HML stock factor is positive, and hence the value premium estimates from stocks and options are not consistent. Several explanations may support the findings. First, Arshanapalli, Fabozzi, and Nelson (2006) show that the value premium can turn statistically negative in stocks in the strong market. The US market from 1996 until 2004 experienced hard times, but still grew about $100 \%$ in these years (if we look at the S\&P100 index). Option buyers may be overestimating the prospects of the economy in comparison to the stock buyers, and hence they may be implicitly pricing the negative value premium in the options. Second, there may be higher replication costs for options on the firms in distress (or in general high value firms), and this leads to lower returns on options. The second point can be verified empirically and we intend to do so in later research.

\footnotetext{
${ }^{11}$ As we have described in section 3.2 , we have 3 moneyness $\times 2$ maturity $\times 2$ option type buckets and all options in them as investable asset classes.
} 


\section{Extensions}

This section discusses how our previous analysis changes if we introduce some real life conditions into our optimization problem ${ }^{12}$. We talk about shortsale constraints and liquidity related issues for stocks, and then also make an investor pay full bid-ask spread while rebalancing derivatives' based factor portfolios.

\subsection{Constrained Optimization}

We assume in our analysis that we have a sophisticated investor, and she has access to all markets and can carry out all possible trades. However, even sophisticated investors will not be able to short the stocks if there are no stocks to borrow. This may happen in the small cap markets or under certain adverse market conditions. If there are options on a stock, one can always take a synthetic forward position to get access to the underlying risk. We investigate how the portfolio composition is going to change from the previous optimization if we impose the shortsale constraints on stocks. As we restrict the investor's assets choice to factor portfolios instead of direct option positions, creating forwards for some stocks may not be possible.

We solve the optimization problem using the same GMM routine as before with an additional condition that the optimal weight of any given stock $j$ at each time $i$ in the portfolio should be non-negative ${ }^{13}$ :

$$
\bar{\omega}_{i, j}+\frac{1}{J_{i}^{s}} \theta^{T} x_{i, j}^{s} \geq 0, \forall i \in\{1, \ldots, 107\}, \forall j\{1, \ldots, 4964\} .
$$

This additional constraint transforms into the formal number of 4,964*107 $=531,148$ inequalities (in reality there are fewer inequalities due to missing returns), and solution is very time consuming. As a consequence we solve the problem only for several interesting factor combinations.

The summary results are provided in Tables 12 - 13. We select for exposition the same combinations as in the unconstrained case, though again we get more significant results. Two points are worth mentioning. First, the stock weights' non-negativity constraints are binding, and the investor now mostly refuses to invest in stock factors except for ME. $\theta$ estimations for BTM and MOM are not significantly different from 0 , while for ME $\theta$ is very alike across all estimated combinations (and significant for log utility case). The $\mathrm{ME}$ factor is dominating the others, and an investor tries to go short the ME factor portfolio. However, the non-negative weight constraints should be stopping her very fast.

\footnotetext{
${ }^{12}$ While we will be mostly talking about the constrained investor problem now, please note that the investor has been constrained in her choice from the very beginning due to an incomplete market. The market is incomplete as we use options as non-redundant assets in addition to the stock market and trying to optimize investor's utility with respect to unknown factors. When we introduce additional frictions, we just reduce the attainable payoff space even more.

${ }^{13}$ The optimization setup is misspecified to some degree here as an investor may not know the value of the characteristic in the remote future. However, as $\theta$ 's are assumed constant over time, we have to impose this forward-looking restriction. Another way to model it would be to optimize with non-negativity constraints assuming today's values of characteristics and later just trim the stock holdings at zero if they get optimally negative.
} 
Second, option-based factors compensate for stocks constraints, and in all but one cases (combination 2 for $\gamma=1.8$, Table 13) we get significant utility improvement over the base constraint case. Moreover, in three cases for each risk aversion level we get a significant utility improvement over the unrestricted base case. Such strategies involve four and more option factor portfolios, and hance may be overstating the utility gains due to transaction costs.

The results for the significance of the option $\theta$ 's estimations resemble to a large extend the unconstraint case: an investor chooses IV-based portfolios, where all estimated $\theta$ 's are significant, while crash factor does not seem to be adding much to the investor's utility. Crash factor $\theta$ 's are significant in combinations with IV factors only, e.g. combination 7 in Tables 12-13. Interestingly, the magnitude of estimated absolute $\theta$ 's is smaller for the constraint case. Two explanations arise: first, this is the way to compensate for the reduced stock factor holdings, i.e. there is an interaction between the stock and options factor portfolios. Second, options are by themselves riskier investment vehicles, and now we invest less in them as we reduced relatively safer stock investment.

In general we can say that option-based factors to a large extend allow an investor to overcome the short-sale constraints. Again, IV based factors prove to be more important than crash factors.

\subsection{Stock Liquidity Factor}

In this section we form the liquidity factor for stocks following Pastor and Stambaugh (2003). They construct liquidity measure that reflects temporary price changes accompanying order flow and find that the aggregate liquidity is a priced state variable in the economy. The liquidity risk is related to the expected return differences that are not explained by stocks' sensitivities to market, size, value, and momentum factors.

We use this approach to study if liquidity is a priced factor in the portfolio with optionbased factors and if options can compensate by its leveraged nature for the liquidity of the underlying stocks. We do not claim, that this is the only way accounting for liquidity in the portfolio optimization, instead we insist that methodology is flexible and allow inclusion of many other factors. We estimate liquidity by the following OLS regression:

$$
r_{i, d+1, j}^{e}=\psi_{i, j}+\phi_{i, j} r_{i, d, j}+\xi_{i, j} \operatorname{sign}\left(r_{i, d, j}^{e}\right) v_{i, d, j}+\epsilon_{i, d+1, j}
$$

$d=1, \ldots, D$, where $r_{i, d, j}$ is the return on stock $j$ on day $d$ in month $i$, and $r_{i, d, j}^{e}$ is the excess return over the market represented by the CRSP value-weighted index, $v_{i, d, j}$ is the dollar trading volume (in millions) for stock $j$ on day $d$ in month $i$. Then the estimated coefficient $\xi_{i, j}$ is the stock's $j$ liquidity in month $i$, and it is calculated only if there are more than 15 return observations in a given month. Same as for the other stocks' and derivatives' characteristics, we standardize $\xi_{i, j}$ and form 3 quantiles at each time $i$.

Intuitively, large stocks are more liquid than small ones. The first inspection of our results confirms this intuition by the correlation of 0.51 between liquidity and size factors (Table 6). Liquidity is also positively correlated with both the value factor (0.18) and momentum (0.19), the latter is in line with the suggestions of Pastor and Stambaugh $(2003)$. 
It is interesting that the estimate of liquidity- $\theta$ appears to be significant in the base case only if the risk aversion coefficient is equal to 1 (log-utility). Including all the factors in our portfolio optimization under the same level of risk aversion does not gain much of the utility from adding liquidity as a factor ( 0.5387 with liquidity and 0.5384 without it).

For the risk aversion of 5 , the significance of the liquidity factor in the portfolio is not high enough, but can be considered in the portfolio if ATM short maturity IV calls factor are included in the the portfolio, sometimes together with the OTM short maturity IV puts factor. Both $\theta$ parameters of this factor portfolios are negative.

The general tendency of the $\theta$ estimates for the factor combinations with liquidity is the same as described in the section (5.2). However, we have to point out that $\theta$ estimates for liquidity are very high for some combination of factors, i.e. our estimates may not be very reliable and including liquidity into the factor combination would need some refinements ${ }^{14}$.

\subsection{Transaction Costs}

Evidently, transaction costs affect the investment policy, and we investigate if investors can adapt knowing that they face bid-ask spread on each rebalancing date. Even for large stocks in S\&P100 index the average bid-ask spread fluctuates between 3\% for deep ITM puts, $7 \%$ for ATM puts and $42 \%$ for deep OTM puts (Horn, Schneider, and Vilkov (2007)), and for small stocks in our sample the rebalancing costs may even be worse.

We approach the problem from two different angles. First, we check what happens to the portfolio performance if the investor optimizes using the mid-price returns and then in reality faces bid-ask spread. Second, we let investor know in advance that each month she will only get the ask-to-bid return on the long options' positions and bid-to-ask return on the short options' positions.

The first approach deteriorates the portfolio performance completely, and this is not surprising with so frequent rebalancing and noted bid-ask spreads. Mean returns on the portfolio vary from $-6.9 \%$ for strategies with only a few ATM option'-based factors to $-745 \%$ in cases when OTM options are added. A cure may be to go for a less frequent rebalancing policy, or no rebalancing at all.

We try another trick: we provide an investor with data on returns assuming real bidask spreads on long and on short positions. Again we assume that an investor liquidates all old positions at the end of a month and creates new ones after that, each time incurring full bid-ask spread. This assumption is not very realistic as part of the last month positions will most probably survive into the next month and no real transaction will take place. We want to see if the options markets remains lucrative under these conditions.

It turns out that an investor is able to adapt partially: her portfolio returns are mostly positive now and range from about $-2 \%$ to $11.54 \%$, but still an investor is not able to attain a higher utility than by just investing in stock based factors. The bottom line is that facing real transaction costs an investor has to optimize not only to risk factors, but also to a rebalancing frequency. However, advanced market participants able to trade at mid market or better can benefit from the analysis in this paper.

\footnotetext{
${ }^{14}$ The table with $\theta$ estimates can be provided upon request.
} 


\section{Conclusion}

Individual stock options represent an important class of financial assets, and investors are looking for ways to identify the trading rules for them. We show empirically that stock option characteristics (such as the implied volatility and the volatility smile skew) contain information on the mispricing in the option portfolios. We build option factor portfolios based on these characteristics, and an individual investor with CRRA utility function can use them to improve her utility. In contrast with the previous research we use a very extensive option prices dataset and directly solve an investor' utility maximization problem.

As a second contribution, we apply a methodology of Brandt, Santa-Clara, and Valkanov (forthcoming) to the portfolio selection with stock options. It allows us to reduce the dimensionality of an investor's optimization problem from the number of assets (which is huge with options and makes optimization unrealistically complex) to a limited number factor/asset class combinations. We form 8 investable asset classes (short and long dated $\times$ OTM and ATM $\times$ calls and puts) for options and show that implied volatility and skew size can be used to build a number of sufficiently independent factor portfolios. Interestingly, IV-based factor portfolios play a much more important role in portfolio formation than the skew size based factors, and in general call option factors are preferable to put factors. Directly estimating the risk premia on the option-based factors in options and stocks, we do not find any significant premia in stock returns, while estimations with the options portfolios do not prove to be robust due to the short sample and very noisy data. The absence of the risk premium on stocks would suggest that we find factors specific to options only, and this issue is left for further research.

When introducing realistic market frictions in the form of shortsale constraints for stocks, we provide evidence that option-based factors can successfully substitute for size, book-to-market and momentum factors. It may be even possible to attain a better utility than in the unconstrained case with stocks only. Again, implied volatility level is an extremely important sorting variable, while skew size plays a secondary role and is mostly an insignificant characteristic.

When an investor faces full bid-ask spread for individual options and has to rebalance fully each month, options lose their lucrativeness and an investor does not want to invest anything in option-based factors. It is true when we apply option returns with bid-ask spread to the strategy without frictions and when we allow an investor to adapt the strategy to real market conditions. Thus, trading the factors identified in this work may be interesting only to market makers and other large market participants that can execute the orders at mid-price or better.

There are several ways to extend the current work. We need to find a better way to estimate the risk premia on the derived factors. We can also try working with different characteristics for options, such as the realized variance difference between the implied and realized measures of variance, or such as some correlation between option returns and macro-variables. Interesting would be to construct a transaction cost factor for derivatives instead of incorporating the bid-ask spread in optimization and to allow investor to optimize with respect to this factor. Finally, we may extend the analysis of the transaction costs for different market participants adjusting the rebalancing frequency or limiting the number of assets rebalanced each period. Those issues are left for future research. 


\section{A Compustat/CRSP Variables}

We use the following items from Compustat (or CRSP if it is given in the brackets) to calculate stock's characteristics:

- price per share - DATA12, DATA13, DATA14 are price-close-1st, 2nd and 3rd month of quarter ('PRC' closing price or bid/ask average),

- shares outstanding - DATA61 common shares outstanding ('SHROUT' shares outstanding),

- total assets - DATA44 assets total,

- liabilities - DATA54 liabilities - total,

- preferred stock value - DATA71 preferred stock redeemable or DATA55 preferred stock carrying value, or 0 ,

- balance sheet deferred taxes - DATA22 deferred taxes \& inv. tax credit,

- monthly return - ('RET' holding period return). 


\section{References}

Ait-Sahalia, Y., and M. W. Brandt, 2001, Variable selection for portfolio choice, Journal of Finance 56, 1297-1351.

Ang, A., R. Hodrick, Y. Xing, and X. Zhang, 2006, Cross-Section of Volatility and Expected Returns, Journal of Finance 61, 259-299.

Arshanapalli, B., F. J. Fabozzi, and W. Nelson, 2006, The value, size, and momentum spread during distressed economic periods, Finance Research Letters 3, 244-252.

Bakshi, G., C. Cao, and Z. Chen, 1997, Empirical Performance of Alternative Option Pricing Models, Journal of Finance 52, 2003-2049.

Bakshi, G., and N. Kapadia, 2003a, Volatility Risk Premium Embedded in Individual Equity Options: Some New Insights, Journal of Derivatives pp. 45-54.

Bakshi, G., and N. Kapadia, 2003b, Delta Hedged Gains and the Negative Market Volatility Risk Premium, Review of Financial Studies pp. 527-566.

Bates, D. S., 1988, Pricing Options on Jump-Diffusion Processes, Rodney L. White Center Working Paper pp. 37-88.

Bates, D. S., 2000, Post-' 87 Crash Fears in the S\&P Futures Option Market, Journal of Econometrics 94, 181-238.

Bates, D. S., 2001, The Market for Crash Risk, NBER Working Papers.

Black, F., and M. Scholes, 1973, The Pricing of Options and Corporate Liabilities, Journal of Political Economy 81, 637-654.

Bondarenko, O., 2003, Why are Put Options So Expensive?, Working Paper.

Brandt, M. W., 1999, Estimating portfolio and consumption choice: A conditional Euler equation approach, Journal of Finance 54, 1609-1646.

Brandt, M. W., P. Santa-Clara, and R. Valkanov, forthcoming, Parametric Portfolio Policies: Exploiting Characteristics in the Cross Section of Equity Returns, Review of Financial Studies.

Branger, N., B. Breuer, and C. Schlag, 2006, Optimal Derivative Strategies with Discrete Rebalancing, Working Paper.

Branger, N., and C. Schlag, 2005, Put Options are not too Expensive. An Analysis of Path Peso Problems, Working Paper.

Carhart, M. M., 1997, On Persistence in Mutual Fund Performance, Journal of Finance $52,57-82$.

Carr, P., and L. Wu, forthcoming, Variance Risk Premia, Review of Financial Studies.

Constantinides, G. M., J. C. Jackwerth, and S. Perrakis, forthcoming, Mispricing of S\&P 500 Index Options, Review of Financial Studies. 
Cox, J., S. Ross, and M. Rubinstein, 1979, Option Pricing: A Simplified Approach, Journal of Financial Economics 7, 229-264.

Daniel, K., and S. Titman, 1997, Evidence on the Characteristics of Cross Sectional Variation in Stock Returns, Journal of Finance 52, 1-33.

Driessen, J., and P. Maenhout, 2007, A portfolio perspective on option pricing anomalies, Review of Finance 11.

Driessen, J., P. Maenhout, and G. Vilkov, 2007, Option-implied Correlations and the Price of Correlation Risk, Working Paper.

Duan, J., and J. Wei, forthcoming, Systematic Risk and the Price Structure of Individual Equity Options, Review of Financial Studies.

Fama, E., and D. MacBeth, 1973, Risk Return and Equilibrium: Empirical Tests, Journal of Financial Political Economy 71, 607-636.

Fama, E. F., and K. R. French, 1992, The cross-section of expected stock returns, Journal of Finance 47, 427-465.

Fama, E. F., and K. R. French, 1996, Multifactor Explanations of Asset Pricing Anomalies, The Journal of Finance 51, 55-84.

Goyal, A., and A. Saretto, 2007, Option Returns and the Cross-Sectional Predictability of Implied Volatility, Working Paper Emory University.

Haugh, M. B., and A. W. Lo, 2001, Asset Allocation and Derivatives, Quantitative Finance $1,45-72$.

Heston, S., 1993, A Closed-Form Solution for Options with Stochastic Volatility with Applications to Bond and Currency Options, Review of Financial Studies p. 327-343.

Horn, D., E. Schneider, and G. Vilkov, 2007, Hedging Options in the Presence of Microstructural Noise, Working Paper.

Jegadeesh, N., and S. Titman, 1993, Returns to Buying Winners and Selling Losers: Implications for Stock Market Efficiency, Journal of Finance 48, 65-91.

Jegadeesh, N., and S. Titman, 2001, Profitability of Momentum Strategies: An Evaluation of Alternative Explanations, Journal of Finance LVI, 699-720.

Jones, C., 1996, A nonlinear factor analysis of S\&P 500 index options returns, Journal of Finance 61, 2325-2363.

Liu, J., and J. Pan, 2003, Dynamic Derivative Strategies, Journal of Financial Economics pp. 401-430.

Merton, R., 1973, Theory of Rational Option Pricing, Bell Journal of Economics pp. $141-183$.

Pastor, L., and R. F. Stambaugh, 2003, Liquidity Risk and Expected Stock Returns, Journal of Political Economy 111, 642-685. 
Pietro, V., and G. Vainberg, 2006, Systematic Variance Risk and Firm Characteristics in the Equity Options Market, Working Paper.

Shanken, J., 1985, Multivariate Tests of Zero-beta CAPM, Journal of Financial Economics $14,327-348$.

Shanken, J., 1992, On the Estimation of Beta Pricing Models, Review of Financial Studies $5,1-34$.

Xing, Y., X. Zhang, and R. Zhao, 2007, What Does Individual Option Volatility Smirks Tell Us about Future Equity Returns?, Working Paper. 


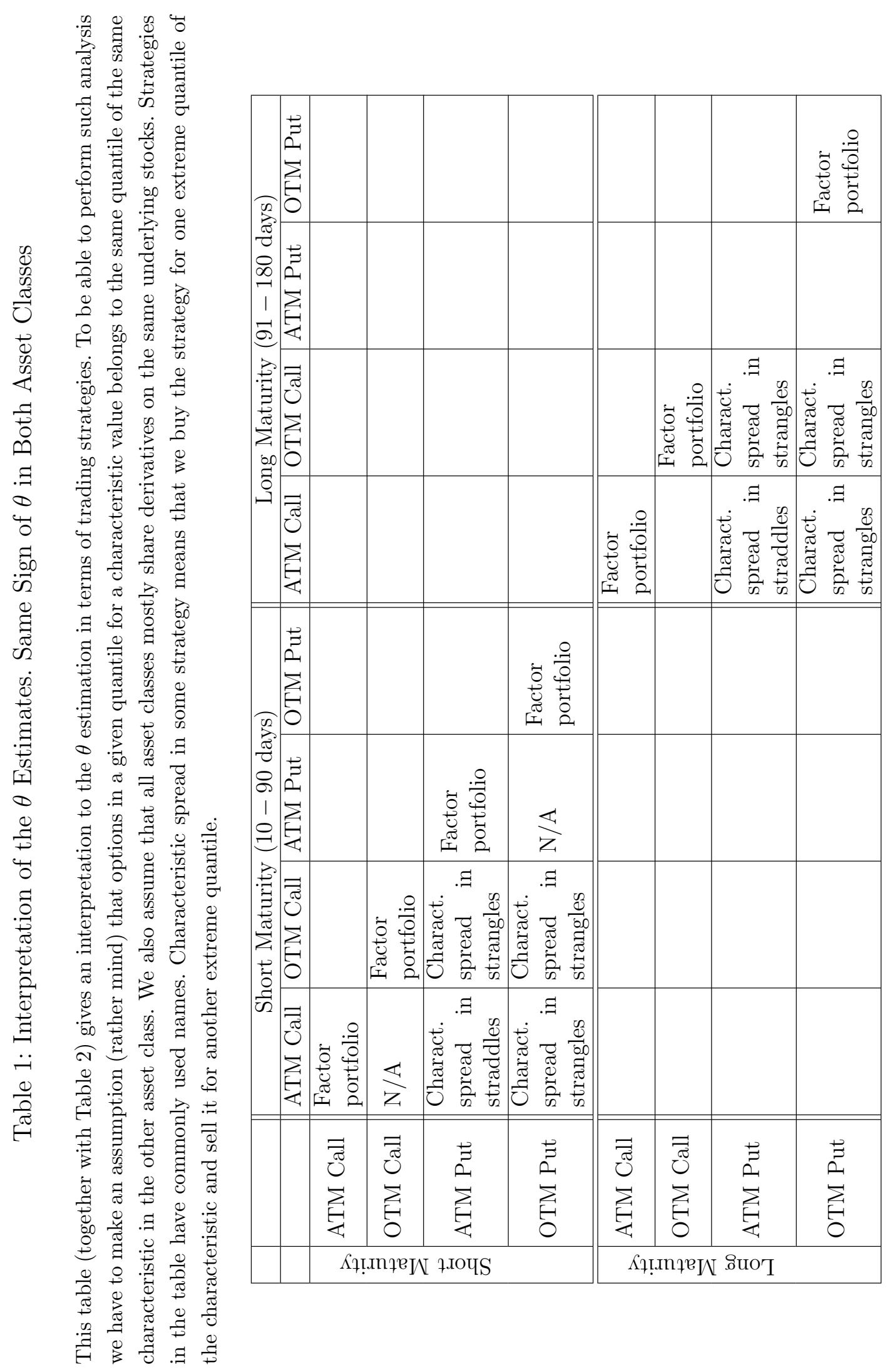




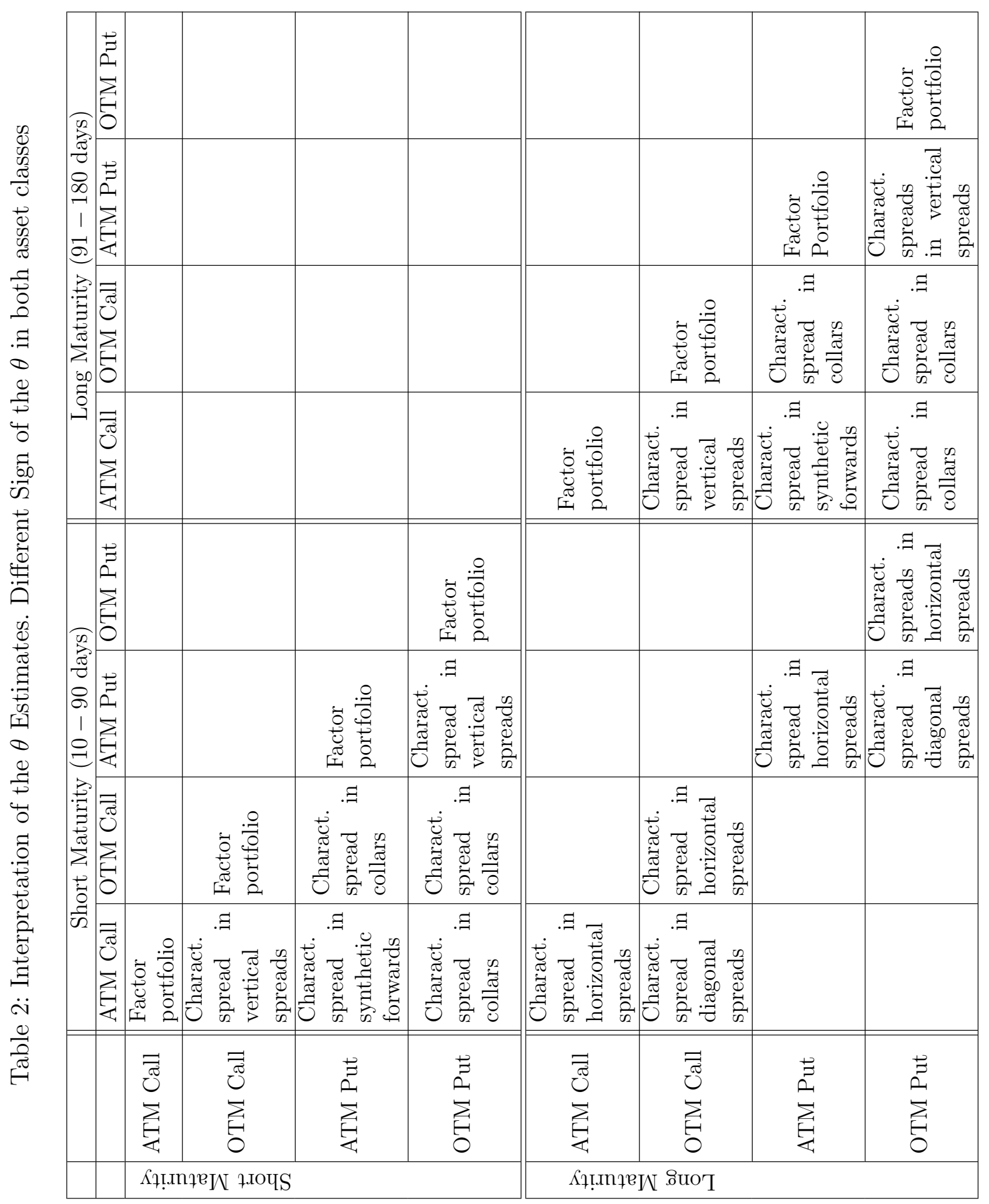


Table 3: Monthly Return Statistics of Call Options for each Bucket

This table gives mean, standard deviation, skew and kurtosis of the return distribution in each call option bucket, where $[0.85 ; 0.95],(0.95 ; 1.05]$ and $(1.05 ; 1.15]$ are moneyness intervals of option buckets. It shows also the average number of calls in the bucket. Each column gives the corresponding parameters for different maturity option: the first are short maturity options (10 to 90 days) and the second are long maturity ones (90 to 180 days).

\begin{tabular}{|c|c|c|}
\hline statistics by moneyness groups & short maturity & long maturity \\
\hline$[\mathbf{0 . 8 5}$; $\mathbf{0 . 9 5}]$ or ITM & 0.0068 & -0.0200 \\
mean & 0.2594 & 0.1695 \\
std. deviation & 1.46 & 1.15 \\
skew & 6.38 & 4.96 \\
kurtosis & 182.11 & 208.47 \\
average number of calls & & \\
(0.95; $\mathbf{1 . 0 5}]$ or ATM & -0.2049 & -0.0695 \\
mean & 0.2556 & 0.2047 \\
std. deviation & 1.21 & 1.10 \\
skew & 4.69 & 4.58 \\
kurtosis & 216.15 & 226.71 \\
average number of calls & & \\
(1.05; $\mathbf{1 . 1 5}$ or OTM & -0.4969 & -0.1923 \\
mean & 0.1591 & 0.2027 \\
std. deviation & 1.13 & 1.22 \\
skew & 4.07 & 5.08 \\
kurtosis & 201.82 & 209.88 \\
\hline
\end{tabular}


Table 4: Monthly Return Statistics of Put Options for each Bucket

This table gives mean, standard deviation, skew and kurtosis of the return distribution in each put option bucket, where $[0.85 ; 0.95],(0.95 ; 1.05]$ and $(1.05 ; 1.15]$ are moneyness intervals of option buckets. It shows also the average number of puts in the bucket. Each column gives the corresponding parameters for different maturity option: the first are short maturity options (10 to 90 days) and the second are long maturity ones (90 to 180 days).

\begin{tabular}{|c|c|c|}
\hline statistics by moneyness groups & short maturity & long maturity \\
\hline$[\mathbf{0 . 8 5}$; $\mathbf{0 5 5}]$ or OTM & -0.5182 & -0.2335 \\
mean & 0.1744 & 0.2030 \\
std. deviation & 2.11 & 1.59 \\
skew & 9.14 & 6.62 \\
kurtosis & 283.01 & 295.78 \\
average number of puts & & \\
(0.95; $\mathbf{1 . 0 5}]$ or ATM & -0.2957 & -0.1417 \\
mean & 0.2504 & 0.1942 \\
std. deviation & 1.76 & 1.58 \\
skew & 7.21 & 6.64 \\
kurtosis & 254.88 & 274.37 \\
average number of puts & & \\
(1.05; $\mathbf{1 . 1 5}]$ or ITM & -0.0557 & -0.0576 \\
mean & 0.2732 & 0.1866 \\
std. deviation & 2.36 & 2.22 \\
skew & 12.59 & 12.04 \\
kurtosis & 162.10 & 201.17 \\
\hline average number of puts &
\end{tabular}


Table 5: Statistics for the Options Factor Portfolios

The Table provides summary statistics for the option factor portfolios. Factor portfolios are constructed from monthly option returns as described in Section 4.2. The Mean and the St.Dev. are given in percent per month, while Sharpe Ratio is annualized.

\begin{tabular}{l|ccccc}
\multicolumn{1}{c|}{ Factors } & Mean & St.Dev. & Skew & Kurtosis & Sharpe Ratio \\
\hline Call OTM Short IV & 6.629 & 19.822 & 0.320 & 2.925 & 1.159 \\
Call OTM Short Skew & 3.326 & 27.154 & 0.493 & 5.148 & 0.424 \\
Call OTM Long IV & 3.408 & 22.094 & -0.221 & 2.897 & 0.534 \\
Call OTM Long Skew & -2.957 & 20.044 & 0.571 & 5.218 & -0.511 \\
Call ATM Short IV & -9.154 & 27.842 & -0.686 & 4.980 & -1.139 \\
Call ATM Short Skew & 3.207 & 34.817 & 0.549 & 7.020 & 0.319 \\
Call ATM Long IV & -8.852 & 25.646 & -0.866 & 3.649 & -1.196 \\
Call ATM Long Skew & 3.921 & 27.091 & -0.340 & 9.888 & 0.501 \\
Put OTM Short IV & 6.305 & 14.426 & 0.160 & 5.192 & 1.514 \\
Put OTM Short Skew & 2.083 & 18.181 & 0.297 & 8.760 & 0.397 \\
Put OTM Long IV & 0.715 & 22.649 & -2.624 & 18.100 & 0.109 \\
Put OTM Long Skew & -0.662 & 14.394 & -1.503 & 11.645 & -0.159 \\
Put ATM Short IV & -1.782 & 20.851 & 0.219 & 3.941 & -0.296 \\
Put ATM Short Skew & -2.878 & 27.184 & -2.954 & 21.134 & -0.367 \\
Put ATM Long IV & -0.385 & 18.610 & -0.331 & 5.254 & -0.072 \\
Put ATM Long Skew & -1.347 & 16.282 & -3.964 & 32.154 & -0.287
\end{tabular}




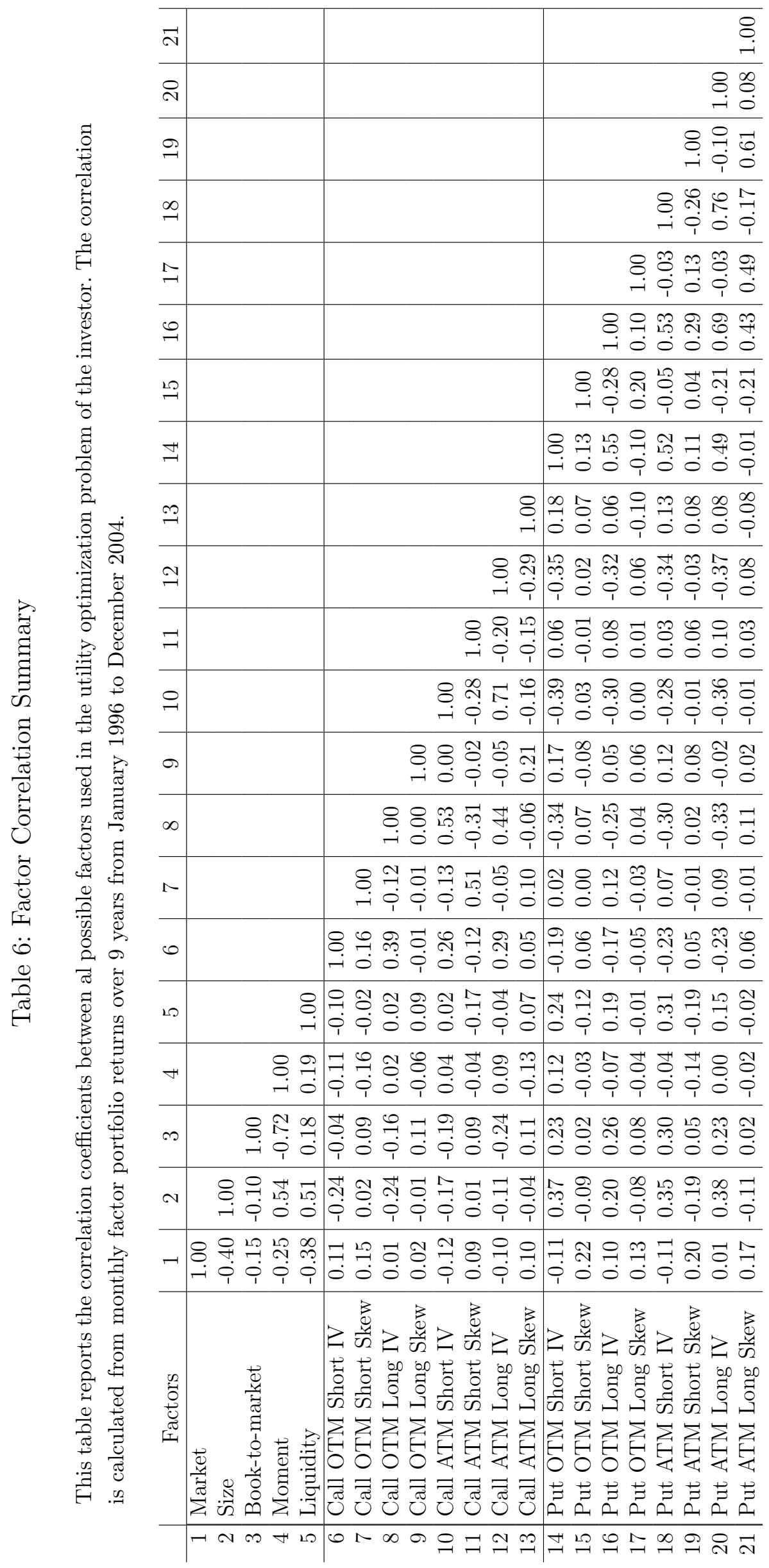


Table 7: $\theta$ Estimates, $\gamma=1$

This Table gives an overview of the portfolio policy estimation of an investor with the $\log$ utility (see Table 8 for the CRRA utility with risk aversion $\gamma=1.8$ ). Each column gives the estimated thetas (bold italic significant at 5\%, and just bold - at $10 \%$ levels) for various factor combinations, and the mean level of utility over all monthly periods with the given thetas combination. Base combination refers to an unconstrained case without option factor portfolios. We calculate the utility improvement for each combination over the base case (to test the significance of the utility difference we use the time series of utilities for each combination pair to get the t-stat of the mean difference). The certainty equivalent shows in percent how much more wealth an investor would require for certain to achieve the same utility as in the current factor combination if she already has the utility as in the base case. Next, the distributional characteristics are calculated from the time series of portfolio returns constructed from optimal thetas and market/ factor monthly returns. The alfas (shown in percent per month) with respect to the three generally accepted models are derived from regressing the portfolio returns on the constant and monthly respective factors.

\begin{tabular}{|c|c|c|c|c|c|c|c|c|c|c|}
\hline Factors & Base & 2 & 3 & 4 & 5 & 6 & 7 & 8 & 9 & 10 \\
\hline Size & -7.70 & -8.20 & -6.39 & -5.00 & -6.59 & -9.13 & -8.20 & -7.39 & -6.49 & -5.43 \\
\hline Book-to-market & 8.01 & 3.66 & 1.86 & -2.29 & 5.33 & 5.43 & 6.60 & -1.00 & 4.32 & -3.51 \\
\hline Moment & 7.64 & 5.68 & 3.41 & 2.68 & 7.23 & 6.75 & 7.16 & 4.87 & 7.03 & 2.05 \\
\hline Call OTM Short IV & - & - & - & 1.47 & 2.19 & - & - & 1.86 & 2.07 & 1.93 \\
\hline Call OTM Short Skew & - & - & - & - & - & - & - & - & - & - \\
\hline Call OTM Long IV & - & - & - & - & - & 1.59 & 2.34 & - & 1.19 & 1.68 \\
\hline Call OTM Long Skew & - & - & - & - & - & - & -1.36 & - & - & - \\
\hline Call ATM Short IV & - & -1.32 & - & - & -1.71 & - & - & -1.49 & -0.73 & -0.83 \\
\hline Call ATM Short Skew & - & - & - & - & - & - & - & - & - & - \\
\hline Call ATM Long IV & - & - & - & - & - & -2.92 & -3.20 & - & -2.12 & -1.57 \\
\hline Call ATM L & - & - & - & - & - & - & 0.70 & - & - & - \\
\hline Put OTM Short IV & - & - & 3.91 & 3.13 & - & - & - & 2.51 & - & 4.45 \\
\hline Put OTM Short Skew & - & - & - & - & - & - & - & - & - & - \\
\hline Put OTM Long IV & - & - & -0.95 & - & - & - & - & - & - & -0.87 \\
\hline Put OTM Long Skew & - & - & - & - & - & - & - & - & - & - \\
\hline Put ATM Shor & - & - & -1.36 & - & - & - & - & 0.01 & - & -1.32 \\
\hline Put ATM Short Skew & - & - & - & - & - & - & - & - & - & - \\
\hline Put ATM Long IV & - & - & 1.60 & - & - & - & - & - & - & 1.32 \\
\hline Put ATM Long Skew & - & - & - & - & _ & - & - & - & - & - \\
\hline Utility whole sample & 0.100 & 0.157 & 0.220 & 0.221 & 0.238 & 0.240 & 0.290 & 0.294 & 0.305 & 0.393 \\
\hline Util diff:comb.-base & 0.000 & 0.058 & 0.120 & 0.121 & 0.138 & 0.141 & 0.190 & 0.194 & 0.206 & 0.294 \\
\hline CE w.r.t. Base & 0.00 & 6.55 & 14.07 & 14.25 & 16.33 & 16.66 & 23.13 & 23.67 & 25.23 & 37.69 \\
\hline Port Return & 18.12 & 29.23 & 38.70 & 39.07 & 45.92 & 50.69 & 61.51 & 56.31 & 58.42 & 79.05 \\
\hline Port Return:std & 39.79 & 52.22 & 52.82 & 55.13 & 66.13 & 82.80 & 96.43 & 72.93 & 78.04 & 90.00 \\
\hline Port Return:skew & 0.222 & 0.283 & -0.117 & -0.025 & 0.160 & 0.865 & 1.394 & 0.205 & 0.742 & 0.208 \\
\hline Port Return:kurtosis & 3.309 & 2.750 & 3.247 & 2.715 & 2.659 & 3.633 & 5.928 & 2.657 & 4.142 & 2.770 \\
\hline Port Return:min & -72.74 & -86.10 & -86.24 & -91.43 & -80.63 & -77.22 & -84.77 & -88.07 & -88.08 & -91.27 \\
\hline Port Retu & 137.57 & 160.38 & 189.83 & 188.32 & 218.87 & 335.77 & 460.14 & 230.45 & 325.19 & 329.75 \\
\hline Port Return:median & 17.58 & 24.99 & 43.24 & 42.09 & 43.52 & 29.93 & 40.76 & 55.22 & 57.84 & 74.94 \\
\hline alfa w.r.t CAPM & 17.49 & 27.02 & 37.38 & 37.23 & 44.05 & 48.29 & 59.37 & 53.60 & 56.23 & 75.98 \\
\hline alfa w.r.t FF & 13.15 & 23.41 & 35.23 & 35.88 & 40.28 & 40.49 & 49.77 & 50.71 & 50.67 & 74.07 \\
\hline alfa w.r.t $\mathrm{FF}+\mathrm{MOM}$ & 12.22 & 22.48 & 32.88 & 32.06 & 38.17 & 40.63 & 49.95 & 46.40 & 48.63 & 70.18 \\
\hline
\end{tabular}


Table 8: $\theta$ Estimates, $\gamma=1.8$

This Table gives an overview of the portfolio policy estimation of an investor with the CRRA utility with risk aversion $\gamma=1.8$ (see Table 7 for $\log$ utility results). Each column gives the estimated thetas (bold italic significant at 5\%, and just bold - at $10 \%$ levels) for various factor combinations, and the mean level of utility over all monthly periods with the given thetas combination. Base combination refers to an unconstrained case without option factor portfolios. We calculate the utility improvement for each combination over the base case (to test the significance of the utility difference we use the time series of utilities for each combination pair to get the t-stat of the mean difference). The certainty equivalent shows in percent how much more wealth an investor would require for certain to achieve the same utility as in the current factor combination if she already has the utility as in the base case. Next, the distributional characteristics are calculated from the time series of portfolio returns constructed from optimal thetas and market/ factor monthly returns. The alfas (shown in percent per month) with respect to the three generally accepted models are derived from regressing the portfolio returns on the constant and monthly respective factors.

\begin{tabular}{|c|c|c|c|c|c|c|c|c|c|c|}
\hline Factors & Base & 2 & 3 & 4 & 5 & 6 & 7 & 8 & 9 & 10 \\
\hline Size & -4.60 & -5.06 & -4.33 & -5.44 & -3.55 & -4.16 & -5.07 & -4.27 & -4.12 & -3.51 \\
\hline Book-to-market & 5.43 & 3.36 & 2.21 & 4.15 & 0.06 & 3.99 & 4.64 & 0.45 & 3.42 & -0.78 \\
\hline Moment & 4.98 & 4.15 & 2.72 & 4.61 & 2.48 & 4.75 & 4.79 & 3.10 & 4.72 & 1.89 \\
\hline Call OTM Short IV & - & - & - & - & 0.96 & 1.38 & - & 1.23 & 1.46 & 1.28 \\
\hline Call OTM Short Skew & - & - & - & - & - & - & - & - & - & - \\
\hline Call OTM Long IV & - & - & - & 1.02 & - & - & 1.49 & - & 0.85 & 1.16 \\
\hline Call OTM Long Skew & - & - & - & - & - & - & -0.97 & - & - & - \\
\hline Call ATM Short IV & - & -0.82 & - & - & - & -1.09 & - & -0.91 & -0.54 & -0.61 \\
\hline Call ATM Short Skew & - & - & - & - & - & - & - & - & - & - \\
\hline Call ATM Long IV & - & - & - & -1.73 & - & - & -1.96 & - & -1.39 & -1.04 \\
\hline Call ATM Long Skew & - & - & - & - & - & - & 0.53 & - & - & - \\
\hline Put OTM Short IV & - & - & 2.56 & - & 2.00 & - & - & 1.77 & - & 2.78 \\
\hline Put OTM Short Skew & - & - & - & - & - & - & - & - & - & - \\
\hline Put OTM Long IV & - & - & -0.67 & - & - & - & - & - & - & -0.57 \\
\hline Put OTM Long Skew & - & - & - & - & - & - & - & - & - & - \\
\hline Put ATM Short IV & - & - & -0.91 & - & - & - & - & -0.17 & - & -0.72 \\
\hline Put ATM Short Skew & - & - & - & - & - & - & - & - & - & - \\
\hline Put ATM Long IV & - & - & 0.98 & - & - & - & - & - & - & 0.66 \\
\hline Put ATM Long Skew & - & - & - & - & - & - & - & - & - & - \\
\hline Utility whole sample & -1.190 & -1.157 & -1.117 & -1.116 & -1.115 & -1.113 & -1.087 & -1.078 & -1.071 & -1.024 \\
\hline Util diff:comb.-base & 0.000 & 0.033 & 0.073 & 0.074 & 0.075 & 0.077 & 0.102 & 0.112 & 0.118 & 0.165 \\
\hline CE w.r.t. Base & 0.00 & 3.77 & 8.72 & 8.87 & 9.03 & 9.25 & 12.65 & 13.95 & 14.89 & 21.88 \\
\hline Port Return:mean & 11.54 & 18.99 & 26.32 & 31.19 & 26.70 & 29.57 & 39.40 & 37.05 & 40.12 & 52.55 \\
\hline Port Return:std & 24.43 & 32.88 & 35.23 & 49.92 & 35.80 & 42.21 & 61.36 & 45.99 & 53.15 & 58.61 \\
\hline Port Return:skew & 0.14 & 0.18 & -0.14 & 0.81 & -0.16 & 0.16 & 1.45 & 0.09 & 0.78 & 0.25 \\
\hline Port Return:kurtosis & 3.28 & 2.67 & 3.32 & 3.44 & 2.66 & 2.65 & 6.16 & 2.68 & 4.32 & 2.94 \\
\hline Port Return:min & -45.30 & -58.79 & -60.00 & -51.22 & -69.51 & -53.59 & -55.99 & -64.68 & -61.08 & -67.94 \\
\hline Port Return:max & 79.70 & 98.26 & 128.47 & 194.93 & 114.02 & 140.27 & 295.33 & 148.74 & 226.00 & 221.27 \\
\hline Port Return:median & 11.86 & 17.76 & 29.83 & 18.75 & 28.70 & 26.86 & 26.54 & 40.20 & 36.09 & 52.78 \\
\hline alfa w.r.t CAPM & 10.97 & 17.55 & 25.33 & 29.64 & 25.43 & 28.17 & 37.87 & 35.24 & 38.46 & 50.53 \\
\hline alfa w.r.t FF & 8.09 & 14.80 & 23.67 & 24.63 & 23.79 & 25.47 & 31.59 & 33.04 & 34.54 & 48.57 \\
\hline alfa w.r.t $\mathrm{FF}+\mathrm{MOM}$ & 7.38 & 14.05 & 22.24 & 24.49 & 21.53 & 24.31 & 31.61 & 30.73 & 33.40 & 46.40 \\
\hline
\end{tabular}



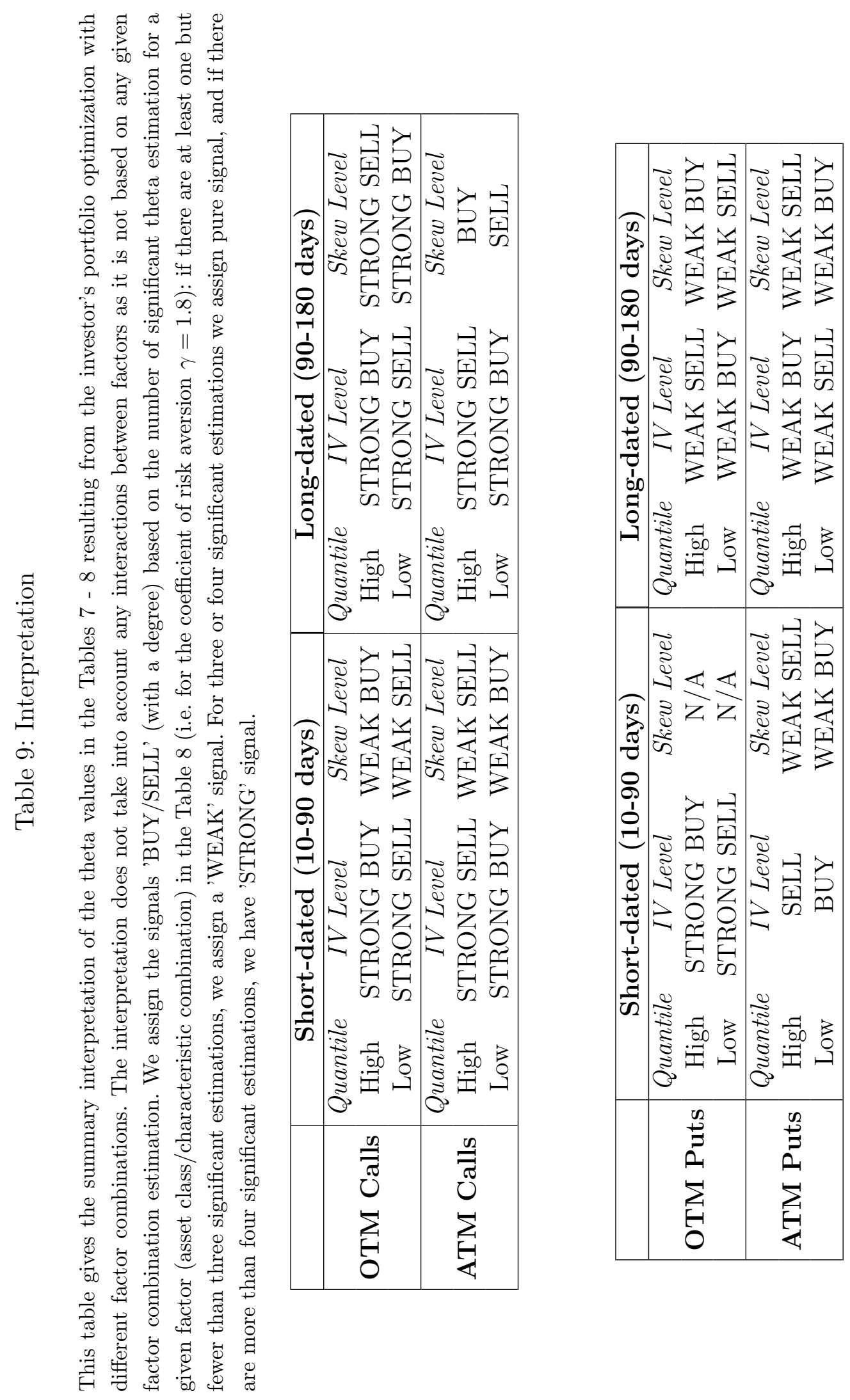
Table 10: Risk Premium Estimated from Option Portfolios

The table shows the estimated risk premia in option returns on five factors: three FamaFrench, momentum and one option-based one. We use time series of 107 monthly returns on 36 option portfolios. We build them by allocating options in each of the option-based asset classes (we regard all options in 3 moneyness $\times 2$ maturity $\times 2$ option type buckets as investable asset classes) to three portfolios using current option-based factor loading $\beta$ as a sorting variable (for the first estimation without the option factor, we sort by market $\beta)$. In the second step we use the GLS regression and adjust the standard errors using the Shanken (1992) correction. Risk premia are in bold italic if significant at $5 \%$ level, and just bold - at $10 \%$ level.

\begin{tabular}{|c|c|c|c|c|c|c|}
\hline Last factor $\backslash$ Factors & Constant & Market & SMB & HML & MOM & Option Factor \\
\hline none & -0.323 & 0.071 & -0.005 & -0.118 & 0.021 & - \\
\hline Call OTM Short IV & -0.226 & 0.027 & 0.020 & -0.014 & -0.045 & 0.322 \\
\hline Call OTM Short Skew & -0.401 & 0.129 & -0.068 & -0.167 & -0.033 & -0.196 \\
\hline Call OTM Long IV & -0.251 & 0.104 & -0.027 & -0.157 & -0.081 & 0.200 \\
\hline Call OTM Long Skew & -0.335 & 0.111 & -0.081 & -0.171 & 0.010 & 0.226 \\
\hline Call ATM Short IV & -0.268 & 0.095 & -0.031 & -0.115 & 0.079 & -0.169 \\
\hline Call ATM Short Skew & -0.326 & 0.055 & -0.054 & -0.105 & 0.050 & -0.128 \\
\hline Call ATM Long IV & -0.295 & 0.103 & 0.023 & -0.183 & -0.003 & -0.027 \\
\hline Call ATM Long Skew & -0.226 & 0.109 & 0.194 & -0.181 & 0.033 & 0.142 \\
\hline Put OTM Short IV & -0.164 & 0.118 & 0.038 & -0.153 & 0.063 & -0.031 \\
\hline Put OTM Short Skew & -0.341 & 0.071 & -0.041 & -0.110 & -0.081 & 0.000 \\
\hline Put OTM Long IV & -0.335 & 0.087 & -0.013 & -0.147 & 0.142 & -0.444 \\
\hline Put OTM Long Skew & -0.294 & 0.188 & 0.076 & -0.281 & 0.045 & -0.244 \\
\hline Put ATM Short IV & -0.274 & 0.073 & 0.042 & -0.130 & 0.111 & -0.482 \\
\hline Put ATM Short Skew & -0.310 & 0.071 & 0.035 & -0.130 & 0.021 & -0.112 \\
\hline Put ATM Long IV & -0.320 & 0.122 & 0.067 & -0.211 & 0.006 & -0.229 \\
\hline Put ATM Long Skew & -0.261 & 0.066 & 0.162 & -0.120 & 0.183 & 0.076 \\
\hline
\end{tabular}


Table 11: Estimated Risk Premium on Option-Based Factors: Summary

The table shows the estimated risk premia in option and stock returns on one option-based factor only (i.e. we show only the last factor statistics from a procedure with 5 factors: three Fama-French, momentum and option-based one). We use time series of 107 monthly returns on 36 option or on 30 stock portfolios. We build option portfolios by allocating options in each of the option-based asset classes to three portfolios using current option-based factor loading $\beta$ as a sorting variable. We build stock portfolios by allocating stocks to 30 portfolios based on the first step regression option-based factor $\beta$ estimates. In the second step we use the GLS regression and adjust the standard errors using the Shanken (1992) correction.

\begin{tabular}{l|cc|cc}
\multicolumn{1}{c|}{ Factor } & $\begin{array}{r}\text { Options } \\
\text { Risk Premium }\end{array}$ & $\begin{array}{r}\text { St-stat } \\
\text { tocks }\end{array}$ \\
\hline Call OTM Short IV & 0.322 & 8.893 & 0.017 & 0.764 \\
Call OTM Short Skew & -0.196 & -1.153 & -0.023 & -0.754 \\
Call OTM Long IV & 0.200 & 2.677 & 0.038 & 1.344 \\
Call OTM Long Skew & 0.226 & 2.265 & 0.017 & 0.774 \\
Call ATM Short IV & -0.169 & -1.622 & 0.030 & 0.857 \\
Call ATM Short Skew & -0.128 & -1.260 & -0.049 & -1.318 \\
Call ATM Long IV & -0.027 & -0.318 & 0.028 & 0.939 \\
Call ATM Long Skew & 0.142 & 1.630 & -0.017 & -0.592 \\
Put OTM Short IV & -0.031 & -0.679 & -0.006 & -0.392 \\
Put OTM Short Skew & 0.000 & -0.005 & -0.011 & -0.525 \\
Put OTM Long IV & -0.444 & -7.307 & -0.006 & -0.230 \\
Put OTM Long Skew & -0.244 & -3.899 & 0.031 & 1.779 \\
Put ATM Short IV & -0.482 & -7.869 & 0.027 & 0.949 \\
Put ATM Short Skew & -0.112 & -1.877 & 0.029 & 0.953 \\
Put ATM Long IV & -0.229 & -4.263 & 0.011 & 0.514 \\
Put ATM Long Skew & 0.076 & 1.964 & 0.014 & 0.758
\end{tabular}


Table 12: $\theta$ Estimates, Constrained Case, $\gamma=1$

This Table gives an overview of the portfolio policy estimation of an investor with log utility (see Table 13 for $\gamma=1.8$ ). Each column gives the estimated thetas (bold italic significant at $5 \%$, and just bold - at $10 \%$ levels) for various factor combinations, and the mean level of utility over all monthly periods for the given thetas combination. Base combination refers to an unconstrained case (Base in Table 7) without option factor portfolios. C.Base combination refers to the constrained optimization problem without option factor portfolios. We calculate the utility improvement for each combination over the base case and over the constrained base case (to test the significance of the utility difference we use the time series of utilities for each combination pair to get the t-stat of the mean difference). The certainty equivalent shows in percent how much more wealth an investor would require for certain each month to achieve the same utility as in the current factor combination if she already has the utility as in the base case. Next, the distributional characteristics are calculated from the time series of portfolio returns constructed from optimal thetas and market/ factor monthly returns. The alfas (shown in percent per month) with respect to the three generally accepted models are derived from regressing the portfolio returns on the constant and monthly respective factors.

\begin{tabular}{|c|c|c|c|c|c|c|c|c|c|c|c|}
\hline Factors & Base & C.Base & 2 & 3 & 4 & 5 & 6 & 7 & 8 & 9 & 10 \\
\hline Size & -7.70 & $\begin{array}{l}-0.09 \\
\end{array}$ & -0.09 & -0.02 & -0.09 & $\begin{array}{c}-0.09 \\
\end{array}$ & $\begin{array}{c}-0.09 \\
\end{array}$ & -0.09 & -0.09 & -0.09 & -0.09 \\
\hline Book-to-market & 8.01 & - & - & - & - & - & - & - & - & - & - \\
\hline Moment & 7.64 & - & - & - & - & - & - & - & - & - & - \\
\hline Call OTM Short IV & - & - & - & - & - & 2.23 & 1.58 & - & 1.73 & 2.96 & 2.10 \\
\hline Call OTM Short Skew & - & - & - & - & - & - & - & - & - & - & - \\
\hline Call OTM Long IV & - & - & - & - & 1.09 & - & - & 2.21 & - & 0.88 & 1.48 \\
\hline Call OTM Long Skew & - & - & - & - & - & - & - & -1.51 & - & - & - \\
\hline Call ATM Short IV & - & - & -1.17 & - & - & -1.44 & - & - & -0.84 & -0.31 & -0.74 \\
\hline Call ATM Short Skew & - & - & - & - & - & - & - & - & - & - & - \\
\hline Call ATM Long IV & - & - & - & - & -1.96 & - & - & -2.62 & - & -2.26 & -1.18 \\
\hline Call ATM Long Skew & - & - & - & - & - & - & - & 0.58 & - & - & - \\
\hline Put OTM Short IV & - & - & - & 2.18 & - & - & 2.47 & - & 2.92 & - & 4.07 \\
\hline Put OTM Short & - & - & - & - & - & - & - & - & - & - & - \\
\hline Put OTM Long IV & - & - & - & -0.19 & - & - & - & - & - & - & -1.01 \\
\hline Put OTM Long Skew & - & - & - & - & - & - & - & - & - & - & - \\
\hline Put ATM Short IV & - & - & - & -0.83 & - & - & - & - & -0.71 & - & -1.97 \\
\hline Put ATM Short Skew & - & - & - & - & - & - & - & - & - & - & - \\
\hline Put ATM Long IV & - & - & - & 0.12 & - & - & - & - & - & - & 1.54 \\
\hline Put ATM Long Skew & - & - & - & - & - & - & - & - & - & - & - \\
\hline Utility whole sample & 0.100 & 0.010 & 0.066 & 0.122 & 0.126 & 0.156 & 0.166 & 0.196 & 0.231 & 0.239 & 0.343 \\
\hline Util.diff:comb.-c.base & -0.090 & 0.000 & 0.056 & 0.112 & 0.116 & 0.146 & 0.156 & 0.186 & 0.221 & 0.229 & 0.334 \\
\hline Util.diff:comb.-base & 0.000 & -0.090 & -0.034 & 0.022 & 0.026 & 0.056 & 0.066 & 0.096 & 0.131 & 0.140 & 0.244 \\
\hline CE wr.t. Base & 9.48 & 0.00 & 5.83 & 11.92 & 12.37 & 15.88 & 17.06 & 20.61 & 24.99 & 26.02 & 39.98 \\
\hline Port Return:mean & 18.12 & 1.14 & 11.85 & 16.03 & 22.21 & 29.10 & 27.12 & 38.61 & 40.03 & 46.71 & 65.17 \\
\hline Port Return:std & 39.79 & 5.28 & 33.49 & 26.09 & 45.94 & 52.62 & 42.87 & 73.43 & 54.67 & 75.25 & 77.64 \\
\hline Port Return:skew & 0.222 & -0.670 & 0.833 & 0.006 & 1.031 & 0.538 & 0.012 & 1.772 & 0.149 & 1.335 & 0.238 \\
\hline Port Return:kurtosis & 3.309 & 3.253 & 4.951 & 3.242 & 5.089 & 3.839 & 2.752 & 7.735 & 3.100 & 6.812 & 2.811 \\
\hline Port Return:min & -72.74 & -16.73 & -77.12 & -47.10 & -84.86 & -82.18 & -91.36 & -88.26 & -92.34 & -88.42 & -90.12 \\
\hline Port Return:max & 137.57 & 9.66 & 149.50 & 96.81 & 188.97 & 197.57 & 126.94 & 356.90 & 203.12 & 386.04 & 287.37 \\
\hline Port Retu & 17.58 & 1.74 & 4.49 & 16.24 & 14.37 & 26.16 & 29.87 & 25.35 & 43.21 & 43.26 & 63.04 \\
\hline alfa w.r.t CAPM & 17.49 & 0.23 & 10.49 & 15.37 & 20.73 & 27.08 & 26.25 & 36.82 & 38.70 & 44.27 & 63.53 \\
\hline alfa w.r.t FF & 13.15 & 0.25 & 9.29 & 15.08 & 17.66 & 26.36 & 24.49 & 32.02 & 36.85 & 41.26 & 62.30 \\
\hline alfa w.r.t $\mathrm{FF}+\mathrm{MOM}$ & 12.22 & 0.33 & 9.22 & 13.78 & 18.19 & 27.30 & 23.72 & 33.02 & 35.79 & 43.19 & 60.91 \\
\hline
\end{tabular}


Table 13: $\theta$ Estimates, Constrained Case, $\gamma=1.8$

This Table gives an overview of the portfolio policy estimation of an investor with CRRA $(\gamma=1.8)$ utility (see Table 12 for the log utility). Each column gives the estimated thetas (bold italic significant at 5\%, and just bold - at 10\% levels) for various factor combinations, and the mean level of utility over all monthly periods for the given thetas combination. Base combination refers to an unconstrained case (Base in Table ??) without option factor portfolios. C.Base combination refers to the constrained optimization problem without option factor portfolios. We calculate the utility improvement for each combination over the base case and over the constrained base case (to test the significance of the utility difference we use the time series of utilities for each combination pair to get the t-stat of the mean difference). The certainty equivalent shows in percent how much more wealth an investor would require for certain each month to achieve the same utility as in the current factor combination if she already has the utility as in the base case. Next, the distributional characteristics are calculated from the time series of portfolio returns constructed from optimal thetas and market/ factor monthly returns. The alfas (shown in percent per month) with respect to the three generally accepted models are derived from regressing the portfolio returns on the constant and monthly respective factors.

\begin{tabular}{|c|c|c|c|c|c|c|c|c|c|c|c|}
\hline Factors & Base & C.Base & 2 & 3 & 4 & 5 & 6 & 7 & 8 & 9 & 10 \\
\hline Size & -4.60 & $\begin{array}{l}-0.09 \\
\end{array}$ & $\begin{array}{c}-0.09 \\
\end{array}$ & -0.09 & -0.09 & -0.09 & -0.09 & -0.09 & -0.09 & -0.09 & $\begin{array}{c}-0.09 \\
\end{array}$ \\
\hline Book-to-market & 5.43 & - & - & - & - & - & - & - & - & - & - \\
\hline Moment & 4.98 & - & - & - & - & - & - & - & - & - & - \\
\hline Call OTM Short IV & - & - & - & - & 1.53 & - & 1.12 & - & 1.82 & 1.27 & 1.35 \\
\hline Call OTM Short Skew & - & - & - & - & - & - & - & - & - & - & - \\
\hline Call OTM Long IV & - & - & - & 1.01 & - & - & - & 1.56 & 0.77 & - & 1.15 \\
\hline Call OTM Long Skew & - & - & - & - & - & - & - & -1.02 & - & - & - \\
\hline Call ATM Short IV & - & - & -0.77 & - & -1.01 & - & - & - & -0.35 & -0.76 & -0.45 \\
\hline Call ATM Short Skew & - & - & - & - & - & - & - & - & - & - & - \\
\hline Call ATM Long IV & - & - & - & -1.47 & - & - & - & -1.86 & -1.45 & - & -1.00 \\
\hline Call ATM Long Skew & - & - & - & - & - & - & - & 0.45 & - & - & - \\
\hline Put OTM Short IV & - & - & - & - & - & 2.81 & 1.79 & - & - & 1.99 & 2.85 \\
\hline Put OTM Short Skew & - & - & - & - & - & 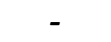 & - & - & - & - & . \\
\hline Put OTM Long IV & - & - & - & - & - & -0.60 & - & - & - & - & -0.64 \\
\hline Put OTM Long Sken & - & - & - & - & - & - & - & - & - & - & - \\
\hline Put ATM Short IV & - & - & - & - & - & -1.04 & - & - & - & -0.64 & -1.14 \\
\hline Put ATM Short Skew & - & - & - & - & - & - & - & - & - & - & - \\
\hline Put ATM Long IV & - & - & - & - & - & 0.46 & - & - & - & - & 0.71 \\
\hline Put ATM Long Skew & - & - & - & - & - & - & - & - & - & - & - \\
\hline Utility whole sample & -1.190 & -1.241 & -1.210 & -1.176 & -1.160 & -1.149 & -1.145 & -1.137 & -1.111 & -1.108 & -1.048 \\
\hline Util.diff:comb.-c.base & 0.052 & 0.000 & 0.032 & 0.065 & 0.082 & 0.092 & 0.096 & 0.104 & 0.130 & 0.133 & 0.194 \\
\hline Util.diff:comb.-base & 0.000 & -0.052 & -0.020 & 0.013 & 0.030 & 0.041 & 0.045 & 0.052 & 0.078 & 0.082 & 0.142 \\
\hline CE w.r.t. base & 5.49 & 0.00 & 3.32 & 6.99 & 8.95 & 10.23 & 10.73 & 11.65 & 14.95 & 15.39 & 23.83 \\
\hline Port Return:mean & 11.54 & 1.14 & 8.17 & 17.63 & 20.58 & 20.13 & 19.82 & 27.65 & 31.89 & 30.23 & 46.27 \\
\hline Port Return:std & 24.43 & 5.28 & 22.51 & 35.29 & 37.05 & 32.58 & 31.01 & 52.29 & 50.01 & 40.91 & 54.23 \\
\hline Port Return:skew & 0.14 & -0.67 & 0.87 & 0.99 & 0.56 & 0.12 & 0.05 & 1.80 & 1.35 & 0.18 & 0.31 \\
\hline Port Return:kurtosis & 3.28 & 3.25 & 4.83 & 5.22 & 3.83 & 3.33 & 2.74 & 7.85 & 6.84 & 3.18 & 3.04 \\
\hline Port Return:min & -45.30 & -16.73 & -49.90 & -70.71 & -57.28 & -54.36 & -64.00 & -61.92 & -71.31 & -72.93 & -66.29 \\
\hline Port Return:max & 79.70 & 9.66 & 100.29 & 142.27 & 139.54 & 124.29 & 93.14 & 256.57 & 253.11 & 151.64 & 211.22 \\
\hline Port Return:median & 11.86 & 1.74 & 3.22 & 11.19 & 18.93 & 20.06 & 20.02 & 17.90 & 28.88 & 31.96 & 44.23 \\
\hline alfa w.r.t CAPM & 10.97 & 0.23 & 6.97 & 16.29 & 18.89 & 19.59 & 18.95 & 26.10 & 29.96 & 28.89 & 44.91 \\
\hline alfa w.r.t FF & 8.09 & 0.25 & 6.18 & 14.08 & 18.38 & 19.72 & 17.67 & 22.67 & 27.94 & 27.73 & 43.69 \\
\hline alfa w.r.t $\mathrm{FF}+\mathrm{MOM}$ & 7.38 & 0.33 & 6.16 & 14.50 & 19.05 & 18.10 & 17.12 & 23.42 & 29.16 & 27.07 & 42.80 \\
\hline
\end{tabular}

